\title{
TOPOLOGICAL LOOPS HAVING SOLVABLE INDECOMPOSABLE LIE GROUPS AS THEIR MULTIPLICATION GROUPS
}

\author{
Á. FIGULA* \\ Institute of Mathematics \\ University of Debrecen \\ H-4002 Debrecen \\ P.O. Box 400, Hungary \\ figula@science.unideb.hu
}

\author{
A. AL-ABAYECHI \\ Institute of Mathematics and \\ Doctoral School of \\ Mathematics and \\ Computational Sciences \\ University of Debrecen \\ H-4002 Debrecen \\ P.O. Box 400, Hungary \\ ameer@science.unideb.hu
}

\begin{abstract}
We prove that the solvability of the multiplication group $\operatorname{Mult}(L)$ of a connected simply connected topological loop $L$ of dimension three forces that $L$ is classically solvable. Moreover, $L$ is congruence solvable if and only if either $L$ has a non-discrete centre or $L$ is an abelian extension of a normal subgroup $\mathbb{R}$ by the 2-dimensional nonabelian Lie group or by an elementary filiform loop. We determine the structure of indecomposable solvable Lie groups which are multiplication groups of three-dimensional topological loops. We find that among the six-dimensional indecomposable solvable Lie groups having a four-dimensional nilradical there are two one-parameter families and a single Lie group which consist of the multiplication groups of the loops $L$. We prove that the corresponding loops are centrally nilpotent of class 2 .
\end{abstract}

\section{Introduction}

The multiplication group $\operatorname{Mult}(L)$ and the inner mapping group $\operatorname{Inn}(L)$ of a loop $L$ are important tools for the investigation of the structure of $L$ since there are strong connections between the structure of the groups $\operatorname{Mult}(L)$ and $\operatorname{Inn}(L)$ and that of $L$. In [B1] R. H. Bruck proved that if the group $\operatorname{Mult}(L)$ is nilpotent, then the loop $L$ is centrally nilpotent and the group $\operatorname{Inn}(L)$ is abelian. In [V] A. Vesanen showed that if the loop $L$ is finite and the group $\operatorname{Mult}(L)$ is solvable, then $L$ is classically solvable; this means there exists a series of subloops of $L$ of the form $\{e\}=L_{0} \leq L_{1} \leq \cdots \leq L_{n}=L$ such that $L_{i-1}$ is a normal subloop in $L_{i}$ and $L_{i} / L_{i-1}$ is an abelian group for all $i=1, \ldots, n$. Since the variety of loops is congruence modular in [SV1], D. Stanovský and P. Vojtěchovský

DOI: $10.1007 /$ s00031-020-09604-1

${ }^{*}$ The paper is supported by the National Research, Development and Innovation Office (NKFIH) Grant No. K132951 and by the EFOP-3.6.1-16-2016-00022 project. The latter project is co-financed by the European Union and the European Social Fund.

Received September 11, 2018. Accepted May 28, 2020.

Published online November 6, 2020.

Corresponding Author: Á. Figula, e-mail: figula@science.unideb.hu 
developed commutator theory for loops following universal algebra. A loop $L$ is called congruence solvable if there is a series $\{e\}=L_{0} \leq L_{1} \leq \cdots \leq L_{n}=L$ of normal subloops of $L$ such that every factor $L_{i} / L_{i-1}$ is abelian in $L / L_{i-1}$. For loops, congruence solvability is strictly stronger than classical solvability (cf. [SV1, Construction 9.1 and Example 9.3, pp. 27, 29] and [F, Exercise 10, pp. 44-45]). In [SV2] D. Stanovský and P. Vojtěchovský proved that a loop $L$ is congruence solvable if and only if it is obtained by iterated abelian extensions.

In [NS1] P. T. Nagy and K. Strambach investigated consistently topological and differentiable loops as topological and differentiable sections in Lie groups. In this paper we follow their treatment and study topological loops $L$ having a solvable Lie group $K$ as their multiplication group. In this case $K$ is a Lie transformation group acting transitively and effectively on the topological space $L$. Using the transitive actions of Lie groups on the space $\mathbb{R}^{n}, n \leq 3$, we show that topological loops $L$ of dimension 3 with solvable multiplication group are classically solvable. In connection to abelian extensions we find, necessary and sufficient condition for $L$ to be congruence solvable (see Theorem 8 ).

The question, which groups can be realized as the multiplication groups and the inner mapping groups of loops motivates a lot of research on loops and their relation to groups (cf. $[\mathrm{C}],[\mathrm{D}],[\mathrm{Ma}],[\mathrm{NV}],[\mathrm{NK} 2])$. The key concept for answering this question is the connected transversals which were introduced by T. Kepka and M. Niemenmaa (cf. [NK1]).

The criteria in [NK1] are applied successfully for Lie groups to be the multiplication groups of topological loops (cf. [F1]-[F5]). In [F1] we proved that only special nilpotent Lie groups, the elementary filiform Lie groups of dimension $\geq 4$, are the multiplication groups of 2-dimensional connected topological proper loops. In [F3] we determined the solvable non-nilpotent connected simply connected Lie groups of dimension $\leq 5$ which are the multiplication groups for 3-dimensional topological loops. Since this classification did not give any example of a topological loop $L$ having an indecomposable solvable Lie group (i.e., a Lie group which is not the direct product of proper connected Lie groups) as the group $\operatorname{Mult}(L)$ of $L$ in the present paper, we turn our attention to this type of group.

In Theorems 9, 11, 12 we give the precise structure of the 3-dimensional connected simply connected loops $L$ and their multiplication groups if $\operatorname{Mult}(L)$ are solvable indecomposable Lie groups. Since the isomorphism classes of the 6-dimensional solvable Lie algebras are fully known (cf. [Mu], [ST], [T]) we applied our results on the one hand for Lie algebras having 2-dimensional centre and on the other hand for those which have 4-dimensional nilradical. We show that the 6dimensional solvable indecomposable Lie groups with one of the following properties:

- they have discrete centre and correspond to 4-dimensional nilradicals,

- they have 1-dimensional centre and belong to 4-dimensional non-abelian nilradicals,

- they have 2-dimensional centre

are not the multiplication groups of 3-dimensional topological loops (cf. Propositions 10 and 14).

In Section 5 we find that among the 6-dimensional solvable indecomposable Lie 
algebras with 1-dimensional centre and 4-dimensional abelian nilradicals there are two classes of Lie algebras depending on a real parameter and a single Lie algebra which consist of the Lie algebras of the multiplication groups of 3-dimensional simply connected topological loops. All these Lie algebras have 3-dimensional abelian commutator subalgebras and their nilradical has an abelian complement in the Lie algebra. We prove that the corresponding loops $L$ are centrally nilpotent of class 2 and determine their inner mapping groups.

Acknowledgements. We thank the referee for the valuable question and Péter T. Nagy for the construction of solvable topological loops which are not congruence solvable.

\section{Preliminaries}

A set $L$ with a binary operation $(x, y) \mapsto x \cdot y$ is called a loop if there exists an element $e \in L$ such that $x=e \cdot x=x \cdot e$ holds for all $x \in L$ and for each $x \in L$ the left translations $\lambda_{x}: L \rightarrow L, \lambda_{x}(y)=x \cdot y$ and the right translations $\rho_{x}: L \rightarrow L$, $\rho_{x}(y)=y \cdot x$ are bijections of $L$. A loop $L$ is proper if it is not a group. The left and right division operations on $L$ are defined by the maps $(x, y) \mapsto x \backslash y=\lambda_{x}^{-1}(y)$, respectively $(x, y) \mapsto y / x=\rho_{x}^{-1}(y), x, y \in L$. Let $\mu_{x}: L \rightarrow L$ be the map $\mu_{x}(y)=$ $y \backslash x$ and hence $\mu_{x}^{-1}(y)=x / y$. The permutation groups $\operatorname{Mult}(L)=\left\langle\lambda_{x}, \rho_{x} ; x \in L\right\rangle$, $T \operatorname{Mult}(L)=\left\langle\lambda_{x}, \rho_{x}, \mu_{x} ; x \in L\right\rangle$ are called the multiplication group and the total multiplication group of $L$. Let $\operatorname{Inn}(L)$, respectively $T \operatorname{Inn}(L)$ be the stabilizer of the identity element $e \in L$ in $\operatorname{Mult}(L)$, respectively in $T \operatorname{Mult}(L)$. They form a subgroup of $\operatorname{Mult}(L)$, respectively in $T \operatorname{Mult}(L)$ and call the inner mapping group, respectively the total inner mapping group of $L$.

The kernel of a homomorphism $\alpha:(L, \cdot) \rightarrow\left(L^{\prime}, *\right)$ of a loop $L$ into a loop $L^{\prime}$ is a normal subloop $N$ of $L$. A word $W$ is a formal product of letters $\lambda_{t(\bar{x})}, \rho_{t(\bar{x})}$ and their inverses, where $t(\bar{x})=t\left(x_{1}, \ldots, x_{n}\right)$ is a loop term. Upon substituting elements $u_{i}$ of a particular loop $L$ for the variables $x_{i}$ in a word $W$ and upon interpreting $\lambda_{t(\bar{x})}, \rho_{t(\bar{x})}$ as translations of $L$, we obtain $W_{\bar{u}}$, an element of $\operatorname{Mult}(L)$. If $W_{\bar{u}}(e)=e$ for every loop $L$ with identity element $e$ and every assignment of elements $u_{i} \in L$ we say that $W$ is an inner word. The concept of tot-inner word is defined similarly allowing $\mu_{t(\bar{x})}$ as generating letters.

The following result describes the commutator of two normal subloops in purely loop theoretical fashion (cf. [SV1]).

Theorem 1. Let $\mathcal{W}$ be a set of tot-inner words such that for every loop $L$ one has $T \operatorname{Inn}(L)=\left\langle W_{\bar{u}}: W \in \mathcal{W}, u_{i} \in L\right\rangle$. Let $L$ be a loop and $N_{1}, N_{2}$ be two normal subloops of $L$. The commutator $\left[N_{1}, N_{2}\right]_{L}$ is the smallest normal subloop of $L$ containing the set $\left\{W_{\bar{u}}(a) / W_{\bar{v}}(a): W \in \mathcal{W}, a \in N_{1}, u_{i}, v_{i} \in L, u_{i} / v_{i} \in N_{2}\right\}$.

Let $T_{x}=\rho_{x}^{-1} \lambda_{x}, U_{x}=\rho_{x}^{-1} \mu_{x}, L_{x, y}=\lambda_{x y}^{-1} \lambda_{x} \lambda_{y}, R_{x, y}=\rho_{y x}^{-1} \rho_{x} \rho_{y}, M_{x, y}=$ $\mu_{y \backslash x}^{-1} \mu_{x} \mu_{y}$. A suitable set of tot-inner words in Theorem 1 is for instance $\mathcal{W}=$ $\left\{T_{x}, U_{x}, L_{x, y}, R_{x, y}, M_{x, y}\right\}$.

A normal subloop $N$ of $L$ is called central, respectively abelian in $L$ if $[N, L]_{L}=$ $\{e\}$, respectively $[N, N]_{L}=\{e\}$. The centre $Z(L)$ of a loop $L$ consists of all elements $z$ which satisfy the equations $z x \cdot y=z \cdot x y, x \cdot y z=x y \cdot z, x z \cdot y=x \cdot z y, z x=x z$ 
for all $x, y \in L$. A normal subloop is central in $L$ if and only if it is a subloop of $Z(L)$. If we put $Z_{0}=e, Z_{1}=Z(L)$ and $Z_{i} / Z_{i-1}=Z\left(L / Z_{i-1}\right)$, then we obtain a series of normal subloops of $L$. If $Z_{n-1}$ is a proper subloop of $L$ but $Z_{n}=L$, then $L$ is centrally nilpotent of class $n$. A loop $L$ is called classically solvable if there is a series $\{e\}=L_{0} \leq L_{1} \leq \cdots \leq L_{n}=L$ of subloops of $L$ such that, for every $i=1,2, \ldots, n, L_{i-1}$ is normal in $L_{i}$ and the factor $L_{i} / L_{i-1}$ is a commutative group. A loop $L$ is said to be congruence solvable if there is a series $\{e\}=L_{0} \leq L_{1} \leq \cdots \leq L_{n}=L$ of normal subloops of $L$ such that every factor loop $L_{i} / L_{i-1}$ is abelian in $L / L_{i-1}$. Every centrally nilpotent loop is congruence solvable. Let $(A,+, 0)$ be a commutative group, let $(F, \cdot, e)$ be a loop, let $\varphi, \phi: F \times F \rightarrow \operatorname{Aut}(A)$ be functions with $\varphi(y, e)=\mathrm{Id}=\phi(e, y)$ and $\theta: F \times F \rightarrow A$ be a function with $\theta(e, y)=0=\theta(y, e)$ for every $y \in F$. The multiplication

$$
(x, a) \oplus(y, b)=(x \cdot y, \varphi(x, y)(a)+\phi(x, y)(b)+\theta(x, y))
$$

defines a loop on $F \times A$, denoted by $L=F \oplus_{\Gamma} A$, which is called the abelian extension of the normal subgroup $A$ by $F$ over the cocycle $\Gamma=(\varphi, \phi, \theta)$. A loop $L$ is called an iterated abelian extension if it has the form

$$
\left.\left(\left(\left(A_{0} \oplus_{\Gamma_{1}} A_{1}\right) \oplus_{\Gamma_{2}} A_{2}\right) \oplus_{\Gamma_{3}} \cdots \oplus_{\Gamma_{k-2}} A_{k-2}\right) \oplus_{\Gamma_{k-1}} A_{k-1}\right) \oplus_{\Gamma_{k}} A_{k}
$$

where $A_{i}, i=0, \ldots, k$, are abelian groups and all extensions are abelian (cf. [SV2], Section 5 and [Mo, Def., p. 380]).

The following assertion is proved in [SV2, Cor. 5.1, p. 380].

Lemma 2. A loop $L$ is congruence solvable if and only if it is an iterated abelian extension.

The next assertion was proved in [A, Thms. 3, 4, and 5], in [B2, IV.1, Lem. 1.3], and in [F5, Lem. 2.3].

Lemma 3. Let $L$ be a loop with multiplication group $\operatorname{Mult}(L)$ and identity elemente.

(i) Let $\alpha$ be a homomorphism of the loop $L$ onto the loop $\alpha(L)$ with kernel $N$. Then $\alpha$ induces a homomorphism of the group $\operatorname{Mult}(L)$ onto the group $\operatorname{Mult}(\alpha(L))$. Let $M(N)$ be the set $\{m \in \operatorname{Mult}(L) ; x N=m(x) N$ for all $x \in$ $L\}$. Then $M(N)$ is a normal subgroup of $\operatorname{Mult}(L)$ containing the multiplication group $\operatorname{Mult}(N)$ of the loop $N$ and the multiplication group of the factor loop $L / N$ is isomorphic to $\operatorname{Mult}(L) / M(N)$.

(ii) For every normal subgroup $\mathcal{N}$ of $\operatorname{Mult}(L)$ the orbit $\mathcal{N}(e)$ is a normal subloop of $L$ and $\mathcal{N} \leq M(\mathcal{N}(e))$.

Let $K$ be a group, let $S \leq K$, and let $A$ and $B$ be two left transversals to $S$ in $K$. We say that $A$ and $B$ are $S$-connected if $a^{-1} b^{-1} a b \in S$ for every $a \in A$ and $b \in B$. The core $\mathrm{Co}_{K}(S)$ of $S$ in $K$ is the largest normal subgroup of $K$ contained in $S$. If $L$ is a loop, then $\Lambda(L)=\left\{\lambda_{a} ; a \in L\right\}$ and $R(L)=\left\{\rho_{a} ; a \in L\right\}$ are $\operatorname{Inn}(L)$ connected transversals in the group $\operatorname{Mult}(L)$. In [NK1] the authors established a purely group theoretical characterization for a group $K$ to be the multiplication group of $L$. 
Lemma 4. A group $K$ is isomorphic to the multiplication group of a loop if and only if there exists a subgroup $S$ with $\mathrm{Co}_{K}(S)=1$ and $S$-connected transversals $A$ and $B$ satisfying $K=\langle A, B\rangle$.

Lemma 5. Let $L$ be a loop with multiplication group $\operatorname{Mult}(L)$ and inner mapping group $\operatorname{Inn}(L)$. Then the normalizer $N_{\operatorname{Mult}(L)}(\operatorname{Inn}(L))$ is the direct product $\operatorname{Inn}(L) \times$ $Z$, where $Z$ is the centre of $\operatorname{Mult}(L)$, and $\operatorname{Co}_{\operatorname{Mult}(L)}(\operatorname{Inn}(L))=\{1\}$.

A loop $L$ is called topological if $L$ is a topological space and the binary operations $(x, y) \mapsto x \cdot y,(x, y) \mapsto x \backslash y,(x, y) \mapsto y / x: L \times L \rightarrow L$ are continuous. In general the multiplication group of $L$ is a topological transformation group that does not have a natural (finite dimensional) differentiable structure. If the multiplication group of $L$ is a Lie group $K$, then $K$ is a Lie transformation group acting transitively and effectively on $L$. Moreover, there is a Lie subgroup $S$ of $K$ with $\operatorname{Co}_{K}(S)=1$ and $S$-connected continuous transversals $A$ and $B$ with $K=\langle A, B\rangle$.

We often use the following lemma. Its first assertion is proved in [H, IX.1], the second assertion is showed in [F2, Lem. 3.3, p. 390].

Lemma 6. For every connected topological loop there exists the universal covering loop $L$. If $L$ is a 3-dimensional connected simply connected topological loop having a solvable Lie group as its multiplication group, then it is homeomorphic to $\mathbb{R}^{3}$.

The elementary filiform Lie group $\mathcal{F}_{n}$ is the simply connected Lie group of dimension $n \geq 3$ such that its Lie algebra has a basis $\left\{e_{1}, \ldots, e_{n}\right\}$ with $\left[e_{1}, e_{i}\right]=$ $e_{i+1}$ for $2 \leq i \leq n-1$. A 2 -dimensional simply connected loop $L_{\mathcal{F}}$ is called an elementary filiform loop if its multiplication group is an elementary filiform group $\mathcal{F}_{n}, n \geq 4$. Every elementary filiform loop is centrally nilpotent of class 2 ([F1, p. 420]). A transitive action of a Lie group $G$ on a manifold $M$ is called primitive, if on $M$ there is no $G$-invariant foliation with connected fibres of positive dimension smaller than $\operatorname{dim} M$. A Lie algebra is called indecomposable, if it is not the direct sum of two proper ideals.

Now we collect the known results about the 3 -dimensional topological loops having solvable Lie groups as their multiplication groups (cf. [F2, Lems. 3.4, 3.5, 3.6 and Props. 3.7, 3.8], [A, pp. 392-393, Thm. 11], [F3, Thm. 6, Sects. 4 and 5], [F5, Props. 2.6, 2.7]).

Lemma 7. Let $L$ be a 3-dimensional proper connected simply connected topological loop such that its multiplication group $\operatorname{Mult}(L)$ is a solvable Lie group.

a) Then the centre $Z$ of the group $\operatorname{Mult}(L)$ and the centre $Z(L)=Z(e)$ of the loop $L$, where $e$ is the identity of $L$, are isomorphic. The centre $Z$ is either discrete or it has dimension 1 or 2 .

b) If $\operatorname{dim}(Z(L))=1$ and the factor loop $L / Z(L)$ is isomorphic to the group $\mathbb{R}^{2}$ or if $\operatorname{dim}(Z(L))=2$, then $L$ is centrally nilpotent of class 2 and the inner mapping group $\operatorname{Inn}(L)$ of $L$ is abelian.

c) If $\operatorname{dim}(Z(L))=2$, then $\operatorname{Mult}(L)$ is a semidirect product of the group $V \cong \mathbb{R}^{m}$, $m \geq 3$, by a group $Q \cong \mathbb{R}$ such that $V=Z \times \operatorname{Inn}(L)$, where $\mathbb{R}^{2}=Z \cong Z(L)$ is the centre of $\operatorname{Mult}(L)$. If $\operatorname{Mult}(L)$ is indecomposable, then for every 1dimensional connected subgroup $N$ of $Z$ the orbit $N(e)$ is a connected central subgroup of $L$ such that the factor loop $L / N(e)$ is not isomorphic to $\mathbb{R}^{2}$. 
d) If $L$ has a 1-dimensional connected normal subloop $N$, then $N$ is isomorphic to the group $\mathbb{R}$ and we have the following possibilities:

(i) The factor loop $L / N$ is isomorphic to $\mathbb{R}^{2}$. Then $N$ is contained in the centre of $L$ and the group $\operatorname{Mult}(L)$ is a semidirect product of the group $P \cong \mathbb{R}^{m}, m \geq 2$ by a group $Q \cong \mathbb{R}^{2}$ such that $P=C \times \operatorname{Inn}(L)$, where $\mathbb{R}=C \cong N$ is a central subgroup of $\operatorname{Mult}(L)$.

(ii) The loop $L / N$ is isomorphic either to the non-abelian 2-dimensional Lie group $\mathcal{L}_{2}$ or to an elementary filiform loop $L_{\mathcal{F}}$. Then the group $\operatorname{Mult}(L)$ has a normal subgroup $S$ containing $\operatorname{Mult}(N) \cong \mathbb{R}$ such that the factor group $\operatorname{Mult}(L) / S$ is isomorphic to the direct product $\mathcal{L}_{2} \times \mathcal{L}_{2}$ if $L / N \cong \mathcal{L}_{2}$ or to an elementary filiform Lie group $\mathcal{F}_{n}, n \geq 4$, if $L / N \cong L_{\mathcal{F}}$.

e) The indecomposable solvable non-nilpotent Lie groups of dimension $\leq 5$ are not the multiplication groups of 3-dimensional topological loops. The centre of every 3-dimensional connected topological proper loop having an at most 6-dimensional indecomposable nilpotent Lie group as its multiplication group has dimension 1.

\section{The structure of indecomposable solvable multiplication groups of 3-dimensional topological loops}

Let $L$ be a 3-dimensional connected simply connected topological proper loop such that its group $\operatorname{Mult}(L)$ is a solvable Lie group. By Lemma 6 the loop $L$ is homeomorphic to $\mathbb{R}^{3}$. The solvable Lie group $\operatorname{Mult}(L)$ has a minimal non-trivial connected normal subgroup $K$ of dimension 1 or 2 . By Lemma 3 the orbit $K(e)$ is a connected normal subloop of $L$. Since the core $\operatorname{Co}_{\operatorname{Mult}(L)}(\operatorname{Inn}(L))$ is trivial one has $K(e) \neq\{e\}$. Hence the dimension of $K(e)$ is 1 or 2. Therefore the group $\operatorname{Mult}(L)$ acts transitively, effectively and imprimitively on the topological space $L$ homeomorphic to $\mathbb{R}^{3}$. According to [L], p. 141, there are three classes of Lie groups $G$ acting imprimitively on $\mathbb{R}^{3}$ :

I. In $\mathbb{R}^{3}$ there is a $G$-invariant foliation $\mathcal{F}$ with 2 -dimensional connected fibres $D$, but there is no $G$-invariant foliation of $D$ with 1-dimensional connected fibres.

II. In $\mathbb{R}^{3}$ there is a $G$-invariant foliation $\mathcal{F}$ with 1 -dimensional connected fibres $C$, but there is no $G$-invariant foliation with 2-dimensional fibres $D$ which are unions of fibres $C$.

III. In $\mathbb{R}^{3}$ there is a $G$-invariant foliation $\mathcal{F}$ with 1 -dimensional connected fibres $C$ and there is a $G$-invariant foliation with 2-dimensional fibres $D$ which are unions of fibres $C$.

If the group $\operatorname{Mult}(L)$ belongs to the I. class, then the loop $L$ has a 2-dimensional connected normal subloop $M$ such that $M$ has no 1-dimensional connected normal subloop. Since $M$ has a Lie group as its multiplication group, $M$ is either a 2dimensional Lie group or an elementary filiform loop. All these loops have a 1dimensional normal subgroup (cf. [F1, p. 420]). This contradiction yields that $\operatorname{Mult}(L)$ is not in the I. class.

If the group $\operatorname{Mult}(L)$ belongs to the II. class, then the loop $L$ has a 1-dimensional connected normal subloop $N$ but there does not exist any 2-dimensional connected normal subloop $M$ of $L$ which contains $N$. Among the Lie algebras acting locally 
primitively on $\mathbb{R}^{2}$ only

$$
\begin{aligned}
& \mathbf{g}_{1}=\langle\partial / \partial x, \partial / \partial y, \alpha(x \partial / \partial x+y \partial / \partial y)+y \partial / \partial x-x \partial / \partial y\rangle, \alpha \geq 0, \text { and } \\
& \mathbf{g}_{2}=\langle\partial / \partial x, \partial / \partial y, x \partial / \partial x+y \partial / \partial y, y \partial / \partial x-x \partial / \partial y\rangle
\end{aligned}
$$

are solvable (cf. [G, p. 341], also [L, Thm. 34, p. 378]). Hence the Lie algebra $\operatorname{mult}(\mathbf{L})$ of $\operatorname{Mult}(L)$ is either isomorphic to one of the Lie algebras $\mathbf{g}_{i}, i=1,2$, or it has a proper subalgebra isomorphic to $\mathbf{g}_{i}, i=1,2$. The first case is impossible since none of the Lie algebras $\mathbf{g}_{i}, i=1,2$, is the Lie algebra of the multiplication group of a 3-dimensional topological loop (cf. [F3, Sect. 4]). In the second case one has

$$
\begin{array}{r}
\operatorname{mult}(\mathbf{L})=\left\langle X_{1}+\phi_{1}(x, y, z) \partial / \partial z, \ldots, X_{k}+\phi_{k}(x, y, z) \partial / \partial z\right. \\
\left.\qquad F_{1}(x, y, z) \partial / \partial z, \ldots, F_{n-k}(x, y, z) \partial / \partial z\right\rangle .
\end{array}
$$

where $X_{1}, \ldots, X_{k}$ are the basis elements of $\mathbf{g}_{i}, i=1,2$, according to whether $\operatorname{mult}(\mathbf{L})$ contains the subalgebra isomorphic to $\mathbf{g}_{i}$. Moreover, the Lie subgroup $A$ of $\operatorname{Mult}(L)$ corresponding to the $(n-k)$-dimensional subalgebra $\mathbf{a}=\left\langle F_{i}(x, y, z) \partial / \partial z\right\rangle$, $i=1, \ldots, n-k$, leaves every 1-dimensional connected left coset $x N, x \in L$, invariant (cf. [L, p. 155]). By Lemma 3 the subgroup $A$ is the normal subgroup $M(N)$ of $\operatorname{Mult}(L)$ and the multiplication group $\operatorname{Mult}(L / N)$ of the 2-dimensional connected factor loop $L / N$ is isomorphic to $\operatorname{Mult}(L) / A$. The factor loop $L / N$ is isomorphic either to a 2-dimensional Lie group or to an elementary filiform loop (cf. Lemma $7 \mathrm{~d})$ ). The factor Lie algebra $\mathbf{m u l t}(\mathbf{L}) / \mathbf{a}$ is isomorphic to $\mathbf{g}_{i}, i=1$ or 2 . But the Lie algebras $\mathbf{g}_{i}, i=1,2$, are not the Lie algebra of the multiplication group of a 2-dimensional topological loop (cf. [F1, Thm. 1, p. 420]).

Hence the group $\operatorname{Mult}(L)$ belongs to the III. class and the loop $L$ has a 2 dimensional connected normal subloop $M$ containing a 1-dimensional connected normal subloop $N$ of $L$. Since $\operatorname{Mult}(L)$ does not belong to the II. class every 1dimensional normal subloop of $L$ lies in a 2-dimensional normal subloop of $L$. By Lemma $7 \mathrm{~d}$ ) the loop $N$ is isomorphic to $\mathbb{R}$ and every orbit of $N$ is homeomorphic to $\mathbb{R}$. By [NS1, Thm. 18.18], the 1-dimensional connected factor loop $L / M$ is isomorphic either to the Lie group $\mathbb{R}$ or to $\mathrm{SO}_{2}(\mathbb{R})$. The normal subloop $M$ and the factor loop $L / N$ are 2-dimensional connected loops having a Lie group as their multiplication groups (cf. Lemma 3). Hence $M$ and $L / N$ are homeomorphic either to $\mathbb{R}^{2}$ or to $S^{1} \times \mathbb{R}$ or to $S^{1} \times S^{1}$ (cf. [NS1, Thm. 19.1, p. 249]). The manifold $L$ is a fibering of $\mathbb{R}^{3}$ over $L / N$ with fibers homeomorphic to $N$ and it is also a fibering of $\mathbb{R}^{3}$ over $L / M$ with fibers homeomorphic to $M$. Hence the first fundamental group $\pi_{1}\left(\mathbb{R}^{3}\right)$ of $\mathbb{R}^{3}$ is isomorphic to the sum $\pi_{1}(L / N)+\pi_{1}(N)$ and also to the sum $\pi_{1}(L / M)+\pi_{1}(M)$. Since $\pi_{1}\left(\mathbb{R}^{n}\right)=0, \pi_{1}\left(S^{1}\right)=\mathbb{Z}$ and $N$ is homeomorphic to $\mathbb{R}$ we obtain that the loops $L / N$ and $M$ are homeomorphic to $\mathbb{R}^{2}$ and $L / M$ is homeomorphic to $\mathbb{R}$. Every 2-dimensional topological loop which is homeomorphic to $\mathbb{R}^{2}$ and having a Lie group as its multiplication group is isomorphic either to an elementary filiform loop or to one of the Lie groups $\left\{\mathbb{R}^{2}, \mathcal{L}_{2}\right\}$ (cf. [F1, Thm. 1]). The series $\{e\}=L_{0} \leq N=L_{1} \leq M=L_{2} \leq L=L_{3}$ of normal subloops of $L$ has the properties that every factor loop $L_{i} / L_{i-1}, i \in\{1,2,3\}$, is isomorphic to $\mathbb{R}$. The above discussion yields case (a) of the following theorem 
Theorem 8. Let $L$ be a proper connected simply connected topological loop of dimension 3 having a solvable Lie group as its multiplication group $\operatorname{Mult}(L)$.

(a) Then $L$ is classically solvable. There is a normal subgroup $N \cong \mathbb{R}$ of $L$. Every normal subgroup $N \cong \mathbb{R}$ of $L$ lies in a 2-dimensional normal subloop $M$ of $L$. The factor loop $L / M$ is isomorphic to $\mathbb{R}$, whereas $M$ and $L / N$ are isomorphic either to a 2-dimensional simply connected Lie group or to an elementary filiform loop.

(b) The loop $L$ is congruence solvable if and only if either $L$ has a non-discrete centre or $L$ is an abelian extension of a 1-dimensional normal subgroup $N \cong$ $\mathbb{R}$ by the factor loop $L / N$ isomorphic either to the Lie group $\mathcal{L}_{2}$ or to an elementary filiform loop $L_{\mathcal{F}}$.

Proof. It remains to prove case (b). By Lemma 2 the loop $L$ is congruence solvable if and only if it is an iterated abelian extension. Among the loops $L$ with solvable multiplication group the following are iterated abelian extensions: If the centre $Z(L)$ of $L$ is non-discrete, then it has dimension 1 or 2 (cf. Lemma 7a)). If $\operatorname{dim}(Z(L))=2$, then $L$ is centrally nilpotent of class 2 (see Lemma $7 \mathrm{~b}$ )) and hence it is congruence solvable. If $Z(L)$ has dimension 1 , then $L$ is an extension of the centre $Z(L) \cong \mathbb{R}$ by a loop $F$ isomorphic to the factor loop $L / Z(L)$. The centre $Z(L)$ is central in $L$ (cf. [SV2, p. 370]) hence it is abelian in $L$. By [SV2, Thm. 4.1, p. 375], the loop $L$ is an abelian extension of $Z(L)$ by $L / Z(L)$. The factor loop $L / Z(L)$ is isomorphic either to $\mathbb{R}^{2}$ or to $\mathcal{L}_{2}$ or to an elementary filiform loop $L_{\mathcal{F}}$ (cf. case (a)). Since $\mathcal{L}_{2}$ is a solvable Lie group and every elementary filiform loop is centrally nilpotent of class 2 the factor loop $L / Z(L)$ is an abelian extension of the group $\mathbb{R}$ by $\mathbb{R}$ (cf. [Mo, Lems. 10, 11, pp. 380-381]). Therefore $L$ is an iterated abelian extension. If the loop $\mathrm{L}$ has a discrete centre, then by case (a) $L$ has a normal subgroup $N \cong \mathbb{R}$ such that the factor loop $L / N$ is isomorphic either to the Lie group $\mathcal{L}_{2}$ or to an elementary filiform loop $L_{\mathcal{F}}$ (Lemma $7 \mathrm{~d}$ )(ii)). Since the factor loop $L / N$ is an abelian extension $L$ is an iterated abelian extension precisely if $L$ is an abelian extension of $N$ by $L / N$.

Schreier's extensions of the normal subgroup $N \cong \mathbb{R}$ by the Lie group $F=\mathcal{L}_{2}$ or by an elementary filiform loop $F=L_{\mathcal{F}}$ are special examples of abelian extensions of $N$ by $F$ (cf. [NS2, p. 761]). Hence they are congruence solvable. Now we give a construction for topological loops which yields non-abelian extensions.

Example 1. Let $(S, \cdot)$ be a topological loop of dimension $n$ having a normal subloop $S_{1}$ such that the factor loop $S / S_{1}$ is isomorphic to the group $\mathbb{R}$ and let $\phi:(S, \cdot) \rightarrow(\mathbb{R},+)$ be a homomorphism. Consider a one-parameter family of loops $\Gamma_{t}: \mathbb{R} \times \mathbb{R} \rightarrow \mathbb{R},(a, b) \mapsto \Gamma_{t}(a, b)=a *_{t} b, t \in \mathbb{R}$, such that $\Gamma_{0}(a, b)=a+b$ and $\Gamma_{t}$ is not commutative for some $t \in \mathbb{R}$. Assume that all loops $\Gamma_{t}$ on the line $\mathbb{R}$ have the same identity element 0 and denote by $\Delta_{t}(a, b): \mathbb{R} \times \mathbb{R} \rightarrow \mathbb{R}$ the right division map $(a, b) \mapsto \Delta_{t}(a, b)=a / t b, t \in \mathbb{R}$, in the loop $\Gamma_{t}$. For the loops $\Gamma_{t}, t \neq 0$, we can take

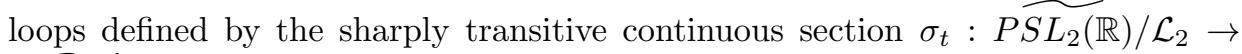
$P L_{2}(\mathbb{R})$ given by the continuous functions $f(u)=\exp \left[\frac{1}{6} \sin ^{2} t \cos u(\cos u-1)\right]$, $g(u)=\left(f(u)^{-1}-f(u)\right) \cot u$ (cf. [NS1, Prop. 18.15 and its proof, pp. 244-245]). All these loops $\Gamma_{t}, t \neq 0$, are proper and hence they are not commutative (cf. Cor. 
18.19., p. 248). The multiplication

$$
(x, a) \circ(y, b)=\left(x \cdot y, \Gamma_{\phi(x \cdot y)}(a, b)\right)
$$

on $S \times \mathbb{R}$ defines a loop $L_{\phi}$ which is an extension of the group $\mathbb{R}$ by the loop $S$. The loop $L_{\phi}$ has the identity element $(1,0)$, where 1 is the identity element of the loop $(S, \cdot)$, because of $(1,0) \circ(y, b)=\left(y, \Gamma_{\phi(y)}(0, b)\right)=(y, b)=(y, b) \circ(1,0)$. Hence the loop $L_{\phi}$ is an Albert extension of the group $\mathbb{R}$ by the loop $(S, \cdot)$ given by the oneparameter family $\Gamma_{t}$ of the loop multiplications on $\mathbb{R}$ (cf. [N, p. 4]). Let $x \in S$ such that $\phi(x) \neq 0$. For the tot-inner word $T(x, a)=\rho_{(x, a)}^{-1} \lambda_{(x, a)}$ one has $T(x, a)(1, c)=$ $((x, a) \circ(1, c)) /(x, a)=\left(x, \Gamma_{\phi(x)}(a, c)\right) /(x, a)=\left(1, \Delta_{\phi(x)}\left(\Gamma_{\phi(x)}(a, c), a\right)\right)$, which is not independent of $a \in \mathbb{R}$ since the loop $\Gamma_{\phi(x)}$ is not commutative. Hence the normal subgroup $\mathbb{R}$ is not abelian in the loop $L_{\phi}$ (cf. [SV2, Proof of Thm. 4.1, p. $377])$. Taking for the loop $(S, \cdot)$ the Lie group $\mathcal{L}_{2}$ or an elementary filiform loop $L_{\mathcal{F}}$ this construction gives a non-abelian extension of the group $\mathbb{R}$ by the loop $(S, \cdot)$.

Theorem 9. Let $L$ be a 3-dimensional proper connected simply connected topological loop such that its multiplication group $\operatorname{Mult}(L)$ is an indecomposable solvable Lie group with 2-dimensional centre $Z$. Then $L$ is centrally nilpotent of class 2 and $\operatorname{Mult}(L)$ has dimension $\geq 6$. The group $\operatorname{Mult}(L)$ is a semidirect product of the subgroup $V=Z \times \operatorname{Inn}(L) \cong \mathbb{R}^{m}, m \geq 5$, by a group $Q \cong \mathbb{R}$, where $\mathbb{R}^{2}=Z \cong Z(L)$. For every 1-dimensional connected subgroup $N$ of $Z$ the orbit $N(e)$ is a connected central subgroup of $L$ and the factor loop $L / N(e)$ is isomorphic to an elementary filiform loop $L_{\mathcal{F}}$. The group $\operatorname{Mult}(L)$ has a normal subgroup $S$ containing $N \cong \mathbb{R}$ such that the factor group $\operatorname{Mult}(L) / S$ is isomorphic to an elementary filiform Lie group $\mathcal{F}_{n}$ with $n \geq 4$.

Proof. According to Lemma 7a),b),c),e), the loop $L$ is centrally nilpotent of class $2, \operatorname{dim}(\operatorname{Mult}(L)) \geq 6$ and $\operatorname{Mult}(L)$ is a semidirect product as in the assertion. Since $N$ is a subgroup of $Z$ the orbit $N(e)$ lies in the centre $Z(L)$ of $L$ and hence $N(e)$ is a 1-dimensional central subgroup of $L$. The multiplication group of the 2-dimensional connected simply connected factor loop $L / N(e)$ is a factor group of $\operatorname{Mult}(L)$. According to Lemma 7c) the loop $L / N(e)$ is not isomorphic to $\mathbb{R}^{2}$. If $L / N(e)$ would be isomorphic to $\mathcal{L}_{2}$, then by Lemma $7 \mathrm{~d}$ )(ii) the group $\operatorname{Mult}(L)$ would have a proper factor group isomorphic to $\mathcal{L}_{2} \times \mathcal{L}_{2}$. A semidirect product $V \rtimes Q$, where $V$ is an abelian normal subgroup of codimension 1 does not have such a factor group. This contradiction yields that $L / N(e)$ is isomorphic to a loop $L_{\mathcal{F}}$ and the remaining part of the assertion follows from Lemma $7 \mathrm{~d}$ )(ii).

Proposition 10. There does not exist any 3-dimensional proper connected topological loop L having a 6-dimensional indecomposable solvable Lie group with 2-dimensional centre as the group $\operatorname{Mult}(L)$ of $L$.

Proof. We may assume that $L$ is simply connected and hence homeomorphic to $\mathbb{R}^{3}$ (cf. Lemma 6). If $\operatorname{Mult}(L)$ is nilpotent, then the assertion follows from Lemma 7 e). According to Theorem 9 the group $\operatorname{Mult}(L)$ has the form $Q \ltimes V$ with the 5-dimensional abelian normal subgroup $V$. Hence the Lie algebra $\operatorname{mult}(\mathbf{L})$ of $\operatorname{Mult}(L)$ has a 5-dimensional abelian nilradical. The unique Lie algebra with 2dimensional centre in the list given in [ST, p. 37], is the Lie algebra $\mathbf{g}_{6,6}$ with 
$a=0=b$ defined by the Lie brackets: $\left[e_{1}, e_{6}\right]=e_{1},\left[e_{3}, e_{6}\right]=e_{2},\left[e_{5}, e_{6}\right]=e_{4}$. The Lie algebra $\mathbf{n}$ of a 1-dimensional central subgroup $N<Z$ has either the form $\mathbf{n}_{\alpha}=\left\langle e_{2}+\alpha e_{4}\right\rangle, \alpha \in \mathbb{R}$, or $\mathbf{n}=\left\langle e_{4}\right\rangle$. There does not exist any ideal $\mathbf{s}$ of $\mathbf{g}_{6,6}$ containing $\mathbf{n}_{\alpha}$ or $\mathbf{n}$ such that the factor algebra $\mathbf{g}_{6,6} / \mathbf{s}$ is isomorphic to an elementary filiform Lie algebra $\mathbf{f}_{n}, n=\{4,5\}$. This contradiction to Theorem 9 proves the assertion.

Theorem 11. Let $L$ be a 3-dimensional proper connected simply connected topological loop having a solvable indecomposable Lie group with a discrete centre as its multiplication group $\operatorname{Mult}(L)$. The loop $L$ has a connected normal subgroup $N$ isomorphic to $\mathbb{R}$ and the factor loop $L / N$ is isomorphic either to the Lie group $\mathcal{L}_{2}$ or to an elementary filiform loop $L_{\mathcal{F}}$. The group $\operatorname{Mult}(L)$ has dimension $\geq 6$ and it has a normal subgroup $S$ containing $\operatorname{Mult}(N) \cong \mathbb{R}$ such that the factor group $\operatorname{Mult}(L) / S$ is isomorphic to the direct product $\mathcal{L}_{2} \times \mathcal{L}_{2}$ if $L / N \cong \mathcal{L}_{2}$ or to an elementary filiform Lie group $\mathcal{F}_{n}, n \geq 4$, if $L / N \cong L_{\mathcal{F}}$. For every 1-dimensional connected normal subgroup $N$ of $L$ the loop $L$ has a normal subloop $M$ isomorphic either to $\mathbb{R}^{2}$ or to $\mathcal{L}_{2}$ or to a loop $L_{\mathcal{F}}$ such that $N$ lies in $M$ and $L / M$ is isomorphic to $\mathbb{R}$. The group $\operatorname{Mult}(L)$ has a normal subgroup $V$ such that the orbit $V(e)$ is the loop $M, \operatorname{Mult}(L) / V \cong \mathbb{R}, V$ contains the inner mapping group $\operatorname{Inn}(L)$ of $L$ and the group $\operatorname{Mult}(M)$ of $M$.

Proof. By Theorem 8(a) there exists a normal subgroup $N$ of $L$ isomorphic to $\mathbb{R}$ and there is a 2-dimensional normal subloop $M$ of $L$ containing $N$. As the group $\operatorname{Mult}(L)$ has a discrete centre the factor loop $L / N$ is not isomorphic to $\mathbb{R}^{2}$ (cf. Lemma 7d)(i)). Hence it is isomorphic either to the Lie group $\mathcal{L}_{2}$ or to a loop $L_{\mathcal{F}}$ and the group $\operatorname{Mult}(L)$ has a normal subgroup $S$ as in the assertion (cf. Lemma $7 \mathrm{~d}$ (ii)). By Lemma 7 e) we have $\operatorname{dim}(\operatorname{Mult}(L)) \geq 6$. Since $L / M$ is isomorphic to $\mathbb{R}$ there is a normal subgroup $V=\{v \in \operatorname{Mult}(L) ; x M=v(x) M$ for all $x \in L\}<$ $\operatorname{Mult}(L)$ such that $V(e)=M, \operatorname{Mult}(L) / V \cong \mathbb{R}$ and $V$ contains the multiplication group $\operatorname{Mult}(M)$ of $M$ (cf. Lemma 3 ). As the group $\operatorname{Mult}(L) / V$ operates sharply transitively on the orbits of $M$ in $L$ the inner mapping group $\operatorname{Inn}(L)$ is a subgroup of $V$.

Theorem 12. Let $L$ be a 3-dimensional proper connected simply connected topological loop such that its multiplication group $\operatorname{Mult}(L)$ is an indecomposable solvable Lie group with 1-dimensional centre $Z$. For every 1-dimensional connected normal subgroup $K$ of $\operatorname{Mult}(L)$ the orbit $K(e)$ is a normal subgroup of $L$ isomorphic to $\mathbb{R}$. We have one of the following possibilities:

(a) The factor loop $L / K(e)$ is isomorphic to $\mathbb{R}^{2}$. Then $L$ is centrally nilpotent of class $2, K(e)$ coincides with the centre $Z(L)$ of $L$ and the group $\operatorname{Mult}(L)$ is a semidirect product of the normal subgroup $P=Z \times \operatorname{Inn}(L) \cong \mathbb{R}^{m}, m \geq 4$, by a group $Q \cong \mathbb{R}^{2}$ and the orbit $P(e)$ is $Z(L)$.

(b) The loop $L / K(e)$ is isomorphic either to the Lie group $\mathcal{L}_{2}$ or to an elementary filiform loop $L_{\mathcal{F}}$. The group $\operatorname{Mult}(L)$ has a normal subgroup $S$ containing $K$ such that the orbit $S(e)$ coincides with $K(e)$, the factor group $\operatorname{Mult}(L) / S$ is isomorphic to the direct product $\mathcal{L}_{2} \times \mathcal{L}_{2}$ if $L / K(e) \cong \mathcal{L}_{2}$ or to an elementary filiform Lie group $\mathcal{F}_{n}, n \geq 4$, if $L / K(e) \cong L_{\mathcal{F}}$. 
The loop $L$ has a 2-dimensional normal subloop $M$ containing $K(e)$ and the group $\operatorname{Mult}(L)$ has a normal subgroup $V$ as in Theorem 11. In particular, if $K(e)=$ $Z(L)$ and $L / Z(L)$ is an elementary filiform loop $L_{\mathcal{F}}$, then $L$ is centrally nilpotent of class 3 and $M$ is not isomorphic to the group $\mathcal{L}_{2}$.

Proof. By Lemmata 3, 5 the normal subloop $K(e)$ is different from $\{e\}$. Applying Lemma 7d) for the case $N=K(e)$ assertion (a) and (b) is proved. By Theorem 8(a) there is a normal subloop $M$ of $L$ containing $K(e)$ and the remaining part of the assertion follows from the proof of Theorem 11. If $K(e)=Z(L)$ and $L / Z(L)$ is an elementary filiform loop $L_{\mathcal{F}}$, then $L$ is an iterated central extension, since every elementary filiform loop is centrally nilpotent of class 2. By [SV2, Cor. 5.2, p. 380], the loop $L$ is centrally nilpotent of class 3 . Moreover, $M$ is not isomorphic to $\mathcal{L}_{2}$ since $\mathcal{L}_{2}$ has a trivial centre.

\section{Six-dimensional indecomposable solvable Lie algebras with four-dimensional nilradical having trivial centre or non-abelian nilradical}

From now on we deal with 6-dimensional indecomposable solvable Lie algebras having 4-dimensional nilradical. Firstly we formulate the main technical tool which we systematically use to exclude those Lie algebras which are not the Lie algebra of the multiplication group of a 3-dimensional topological loop.

Proposition 13. Let $L$ be a 3-dimensional connected simply connected topological loop having a 6-dimensional solvable indecomposable Lie algebra $\mathbf{g}$ with 4-dimensional nilradical $\mathbf{n}_{\mathrm{rad}}$ as the Lie algebra of its multiplication group.

a) For each 1-dimensional ideal $\mathbf{i}$ of $\mathbf{g}$ the orbit $I(e)$, where $I$ is the simply connected Lie group of $\mathbf{i}$ and $e$ is the identity element of $L$, is a normal subgroup of $L$ isomorphic to $\mathbb{R}$. We have one of the following possibilities:

(i) The factor loop $L / I(e)$ is isomorphic to $\mathbb{R}^{2}$ and for the nilradical one has $\mathbf{n}_{\text {rad }}=\mathbf{z} \oplus \mathbf{i n n}(\mathbf{L}) \cong \mathbb{R}^{4}$, where $\mathbf{z}$ is the 1-dimensional centre of $\mathbf{g}$ and $\operatorname{inn}(\mathbf{L})$ is the Lie algebra of the group $\operatorname{Inn}(L)$.

(ii) The factor loop $L / I(e)$ is isomorphic either to the Lie group $\mathcal{L}_{2}$ or to an elementary filiform loop $L_{\mathcal{F}}$. Then there exists a 2-dimensional ideal $\mathbf{s}$ of $\mathbf{g}$ such that $\mathbf{i}<\mathbf{s}$ and the factor Lie algebra $\mathbf{g} / \mathbf{s}$ is isomorphic either to the direct sum $\mathbf{l}_{2} \oplus \mathbf{l}_{2}$, where $\mathbf{l}_{2}$ is the 2-dimensional non-abelian Lie algebra or to the elementary filiform Lie algebra $\mathbf{f}_{4}$.

Assume that the centre of $\mathbf{g}$ is trivial or the nilradical of $\mathbf{g}$ is not abelian.

b) For every 2-dimensional abelian ideal a of $\mathbf{g}$ such that the factor Lie algebra $\mathbf{g} / \mathbf{a}$ is isomorphic neither to $\mathbf{l}_{2} \oplus \mathbf{l}_{2}$ nor to $\mathbf{f}_{4}$ and for each nilpotent ideal $\mathbf{s}$ of $\mathbf{g}$ having dimension $>2$ the orbits $A(e), S(e)$, where $A$, respectively $S$ $i s$ the simply connected Lie group of $\mathbf{a}$, respectively $\mathbf{s}$ and $e$ is the identity element of $L$, are the same 2-dimensional normal subloop $M$ of $L$. There exists a 5-dimensional ideal $\mathbf{v}$ of $\mathbf{g}$ containing the Lie algebra $\mathbf{i n n}(\mathbf{L})$, the Lie algebra $\operatorname{mult}(M)$ of the multiplication group of $M$ and the nilradical $\mathbf{n}_{\mathrm{rad}}$. For every ideal $\mathbf{a}$, respectively $\mathbf{s}$ one has $\mathbf{a} \cap \mathbf{i n n}(\mathbf{L})=\{0\}$ and the intersection $\mathbf{s} \cap \operatorname{inn}(\mathbf{L})$ has dimension $\operatorname{dim}(\mathbf{s})-2$. In particular, if $\mathbf{g}$ is not the Lie algebra $N_{6,28}$ in [T, Table III, p. 1349], then the loop $M$ is isomorphic to $\mathbb{R}^{2}$. 
Proof. Each 1-dimensional ideal $\mathbf{i}$ of $\mathbf{g}$ lies in $\mathbf{n}_{\text {rad }}$, which is isomorphic either to $\mathbb{R}^{4}$ or to $\mathbf{f}_{3} \oplus \mathbb{R}$ or to $\mathbf{f}_{4}$ (cf. [T]). If the factor loop $L / I(e)$ is isomorphic to $\mathbb{R}^{2}$, then the orbit $I(e)$ coincides with the 1-dimensional centre $Z(L)$ of $L$ (cf. Proposition 10 and Theorem 12). The Lie algebra $\mathbf{p}$ of the normal subgroup $P$ in Theorem 12(a) is a 4-dimensional abelian ideal $\mathbf{p}=\mathbf{z} \oplus \mathbf{i n n}(\mathbf{L})$ of $\mathbf{g}$ such that the commutator ideal $\mathbf{g}^{\prime}$ is contained in $\mathbf{p}$. The ideal $\mathbf{p}$ is nilpotent hence it coincides with the nilradical $\mathbf{n}_{\text {rad }}$ of $\mathbf{g}$. This proves assertion (i). Since $\mathbf{g}$ has no factor Lie algebra isomorphic to the 5 -dimensional elementary filiform Lie algebra $\mathbf{f}_{5}$ assertion (ii) follows from Theorems 11, 12(b).

Assume that the centre of $\mathbf{g}$ is trivial or the nilradical of $\mathbf{g}$ is not abelian. Taking into account [T, Tables I, III, IV, V, pp. 1347-1350], the commutator Lie algebra $\mathbf{g}^{\prime}$ of $\mathbf{g}$ coincides with the nilradical $\mathbf{n}_{\text {rad }}$ of $\mathbf{g}$. Let $\mathbf{a}$ be a 2 -dimensional abelian ideal of $\mathbf{g}$. The orbit $A(e)$ is a normal subloop of $L$ (cf. Lemma 3) such that $A(e) \neq\{e\}$ and $\operatorname{dim}(A(e)) \neq 1$ (cf. Lemmata 5, $7 \mathrm{~d}$ ) and Proposition $13 \mathrm{a}$ ). Hence $A(e)$ is a 2-dimensional normal subloop $M$ of $L$. The 5-dimensional ideal $\mathbf{v}$ is the Lie algebra of the normal subgroup $V$ in Theorem 11 and hence one has $V(e)=M$. The ideal a is contained in $\mathbf{n}_{\mathrm{rad}}$. Let $N$ be the simply connected Lie group of $\mathbf{n}_{\text {rad }}$. The orbit $N(e)$ is a normal subloop of $L$ having dimension 2 or 3 since $A(e) \subseteq N(e)$. Therefore $N(e)$ is either the subloop $M$ or the loop $L$. As $\mathbf{g}^{\prime}=\mathbf{n}_{\text {rad }}$ one has $\mathbf{n}_{\text {rad }} \subset \mathbf{v}$. Hence we obtain that $V(e)=N(e)=A(e):=M$. Since each nilpotent ideal $\mathbf{a}$ and $\mathbf{s}$ in assertion b) is contained in $\mathbf{n}_{\mathrm{rad}}$ one has $A(e)=S(e)=N(e)=M$. Since $\operatorname{dim}(A(e))=2$ the 2-dimensional abelian Lie group $A$ acts sharply transitively on $A(e)$. Hence one has $A \cap \operatorname{Inn}(L)=\{1\}$. As $\operatorname{dim}(\mathbf{s})>2$ and $\operatorname{dim}(S(e))=2$ there is a subgroup of $S$ of $\operatorname{dimension} \operatorname{dim}(\mathbf{s})-2$, which fixes the identity element $e$ of $L$. The ideal $\mathbf{v}$ contains the Lie algebra $\operatorname{mult}(M)$ of the group $\operatorname{Mult}(M)$ of the 2-dimensional normal subloop $M$ of $L$. The loop $M$ is isomorphic either to $\mathbb{R}^{2}$ or to $\mathcal{L}_{2}$ or to a loop $L_{\mathcal{F}}$ (cf. Theorem 11). Since $\mathbf{n}_{\text {rad }} \subset \mathbf{v}$ and $\operatorname{dim}(\mathbf{v})=5$ the intersection of $\mathbf{v}$ with the complement of $\mathbf{n}_{\text {rad }}$ in $\mathbf{g}$ has dimension 1 . Therefore $\mathbf{v}$ does not contain a subalgebra isomorphic to $\mathbf{l}_{2} \oplus \mathbf{l}_{2}$. The radical of the Lie algebras $\mathbf{g}$ which are different from $N_{6,28}$ does not contain an elementary filiform Lie algebra of dimension $\geq 4$. Hence one has $M=V(e)=\mathbb{R}^{2}$. This proves assertion b).

Now we prove that the 6 -dimensional solvable indecomposable Lie algebras with 4-dimensional nilradical having trivial centre or non-abelian nilradical are not the Lie algebras of the multiplication groups of 3-dimensional topological loops.

Proposition 14. Let $\mathbf{g}$ be a 6-dimensional solvable indecomposable Lie algebra with 4-dimensional nilradical $\mathbf{n}_{\mathrm{rad}}$ such that $\mathbf{g}$ has trivial centre or $\mathbf{n}_{\mathrm{rad}}$ is not abelian. There does not exist a 3-dimensional connected topological loop L having $\mathrm{g}$ as the Lie algebra of the multiplication group $\operatorname{Mult}(L)$ of $L$.

Proof. We may assume that $L$ is simply connected and hence it is homeomorphic to $\mathbb{R}^{3}$ (cf. Lemma 6). The 6-dimensional solvable indecomposable Lie algebras with 4-dimensional nilradical having trivial centre or non-abelian nilradical are listed $[\mathrm{T}$, Tables I, III, IV, V, pp. 1347-1350]. The Lie algebras $N_{6, i}, i=4,7,30,39,40$, have the ideal $\mathbf{i}=\left\langle n_{4}\right\rangle$. The Lie algebras $N_{6, i}, i=5,16,17$, have the ideal $\mathbf{i}=\left\langle n_{2}\right\rangle$. The Lie algebras $N_{6, i}, i=8,9,10,13,14,28,35,36,37$, have the ideal $\mathbf{i}=\left\langle n_{1}\right\rangle$. 
There does not exist any ideal $\mathbf{s}$ of the above Lie algebras $N_{6, i}$ which contains $\mathbf{i}$ and the factor Lie algebras $N_{6, i} / \mathbf{s}$ are isomorphic either to $\mathbf{f}_{4}$ or to $\mathbf{l}_{2} \oplus \mathbf{l}_{2}$. For $i=39,40$, the nilradical of $N_{6, i}$ is not abelian. Hence the factor loop $L / I(e)$ is not isomorphic to $\mathbb{R}^{2}$. By Proposition 13a)(i),(ii) these Lie algebras are not the Lie algebras of the multiplication groups of 3-dimensional topological loops. The Lie algebras $N_{6, j}, j=12,15,18,19$, have no 1-dimensional ideal. The unique 2dimensional abelian ideal of $N_{6,12}$, respectively $N_{6,19}$ is $\mathbf{s}_{1}=\left\langle n_{2}, n_{4}\right\rangle$, respectively $\mathbf{s}_{2}=\left\langle n_{3}, n_{4}\right\rangle$. The Lie algebras $N_{6,15}, N_{6,18}$ have two 2-dimensional abelian ideals $\mathbf{s}_{2}$ and $\mathbf{s}_{3}=\left\langle n_{1}, n_{2}\right\rangle$. None of the factor algebras $N_{6,12} / \mathbf{s}_{1}, N_{6,19} / \mathbf{s}_{2}, N_{6, j} / \mathbf{s}_{k}$, $j=15,18, k=2,3$, are isomorphic to $\mathbf{f}_{4}$ or to $\mathbf{l}_{2} \oplus \mathbf{l}_{2}$. Hence the orbits $S_{i}(e)$, where $S_{i}=\exp \left(\mathbf{s}_{\mathbf{i}}\right), i=1,2,3$, are 2-dimensional normal subloops of $L$ (cf. Proposition 13b)). If $N_{6, j}, j=12,15,18,19$, would be the Lie algebra of the multiplication group of a 3-dimensional topological loop, then $L$ would have no 1-dimensional normal subgroup. This contradiction to Theorem 8 (a) excludes these Lie algebras.

The Lie algebras $N_{6, i}, i \in\{1,2,3,6,11\}$, have trivial centre and neither a subalgebra nor a factor Lie algebra are isomorphic to an elementary filiform Lie algebra. The Lie algebra $N_{6,1}$ depends on four real parameters $\alpha, \beta, \gamma, \delta$ with $\alpha \beta \neq 0, \gamma^{2}+\delta^{2} \neq 0$. It has the ideals $\mathbf{i}_{1}=\left\langle n_{3}\right\rangle, \mathbf{i}_{2}=\left\langle n_{4}\right\rangle$. If $N_{6,1}$ is the Lie algebra of the multiplication group of a 3 -dimensional topological loop, then there are 2-dimensional ideals $\mathbf{s}_{j}$ of $N_{6,1}$ containing $\mathbf{i}_{j}, j=1,2$, such that the factor Lie algebras $N_{6,1} / \mathbf{s}_{j}, j=1,2$, are isomorphic to $\mathbf{l}_{2} \oplus \mathbf{l}_{2}$ (cf. Theorem 11 and Proposition 13a)(ii). This is the case if and only if $\gamma=\delta=0$. This contradiction excludes the Lie algebra $N_{6,1}$.

The Lie algebra $N_{6,2}$ depends on three real parameters $\alpha, \beta, \gamma$ and the Lie algebra $N_{6,6}$ depends on $\alpha, \beta$ such that in both cases one has $\alpha^{2}+\beta^{2} \neq 0$. The Lie algebras $N_{6,3}, N_{6,11}$ depend on the real parameter $\alpha$. The Lie algebra $N_{6,2}$ has the ideals $\mathbf{i}_{1}=\left\langle n_{1}\right\rangle, \mathbf{i}_{2}=\left\langle n_{2}\right\rangle, \mathbf{i}_{3}=\left\langle n_{4}\right\rangle$ and the Lie algebras $N_{6, j}, j=3,6,11$, have the ideals $\mathbf{i}_{k}, k=2,3$. If $N_{6, j}, j=2,3,6,11$, would be the Lie algebra of the multiplication group of a 3-dimensional topological loop, then applying Theorem 11 and Proposition 13a)(ii) there are 2-dimensional ideals $\mathbf{s}$ of $N_{6, j}, j=2,3,6,11$, containing $\mathbf{i}_{k}, k=1,2,3$, such that the factor Lie algebras $N_{6, j} / \mathbf{s}, j=2,3,6,11$, are isomorphic to $\mathbf{l}_{2} \oplus \mathbf{l}_{2}$. For the ideals $\mathbf{s}_{1}=\left\langle n_{1}, n_{4}\right\rangle, \mathbf{s}_{2}=\left\langle n_{2}, n_{4}\right\rangle$ of $N_{6,2}$, respectively for the ideal $\mathbf{s}_{2}$ of the Lie algebras $N_{6, j}, j=3,6,11$, the factor Lie algebras $N_{6,2} / \mathbf{s}_{i}$, $i=1,2$, respectively $N_{6, j} / \mathbf{s}_{2}, j=3,6,11$, are isomorphic to $\mathbf{l}_{2} \oplus \mathbf{l}_{2}$ precisely if $\beta=\gamma=0$, respectively $\alpha=0$. Hence we have to consider the Lie algebras $N_{6,2}$ with $\beta=\gamma=0, \alpha \neq 0, N_{6, j}, j=3,11$, with $\alpha=0$ and $N_{6,6}$ with $\alpha=0$, $\beta \neq 0$. These Lie algebras have the abelian ideals $\mathbf{s}_{3}=\left\langle n_{1}, n_{2}\right\rangle, \mathbf{s}_{4}=\left\langle n_{3}, n_{4}\right\rangle$ such that the factor Lie algebras $N_{6, j} / \mathbf{s}_{l}, j=2,3,6,11, l=3,4$, are not isomorphic to $\mathbf{l}_{2} \oplus \mathbf{l}_{2}$. The 3 -dimensional abelian ideals $\mathbf{s}_{5}=\left\langle n_{1}, n_{2}, n_{4}\right\rangle, \mathbf{s}_{6}=\left\langle n_{2}, n_{3}, n_{4}\right\rangle$, $\mathbf{s}_{7}=\left\langle n_{1}, n_{3}, n_{4}\right\rangle$ of $N_{6,2}$ and the ideals $\mathbf{s}_{m}, m=5,6$, of $N_{6, j}, j=3,6,11$, are in $\mathbf{n}_{\text {rad }}$. According to Proposition $\left.13 \mathrm{~b}\right)$ the orbits $S_{l}(e)$, where $S_{l}=\exp \left(\mathbf{s}_{\mathbf{l}}\right)$, $l \in\{3,4,5,6,7\}$, and the orbit $N(e)$, where $N$ is the simply connected Lie group of $\mathbf{n}_{\text {rad }}$, are the same normal subgroup $M \cong \mathbb{R}^{2}$ of $L$. Since $\mathbf{i}_{k} \subset \mathbf{n}_{\text {rad }}, k=1,2,3$, the group $M$ contains the 1-dimensional normal subgroups $I_{k}(e)$ of $L$, where $I_{k}$ are the simply connected Lie groups of $\mathbf{i}_{k}, k \in\{1,2,3\}$. The ideal $\mathbf{v}$ in Proposition $13 \mathrm{~b}$ ) has one of the following forms: $\mathbf{v}_{1, k}=\left\langle n_{1}, n_{2}, n_{3}, n_{4}, x_{1}+k x_{2}\right\rangle, k \in \mathbb{R}$, 
$\mathbf{v}_{2}=\left\langle n_{1}, n_{2}, n_{3}, n_{4}, x_{2}\right\rangle$. For $l=3,4$ one has $\mathbf{s}_{l} \cap \mathbf{i n n}(\mathbf{L})=\{0\}$, for $m=5,6,7$ the intersections $\mathbf{s}_{m} \cap \operatorname{inn}(\mathbf{L})$ have dimension 1 and $\operatorname{dim}\left(\mathbf{n}_{\mathrm{rad}} \cap \operatorname{inn}(\mathbf{L})\right)=2$. Hence the Lie subalgebra $\operatorname{inn}(\mathbf{L})$ of $N_{6, j}, j=2,3,6,11$, has either the basis elements $b_{1}=n_{2}+a_{1} n_{4}, b_{2}=n_{1}+a_{2} n_{3}+a_{4} n_{4}$, where $a_{1} a_{2} \neq 0$ or the basis elements $b_{1}^{\prime}=n_{1}+a_{1} n_{2}+a_{2} n_{4}, b_{2}^{\prime}=n_{2}+a_{3} n_{3}+a_{4} n_{4}$, where $a_{2} a_{3} \neq 0$. In the second case for the Lie algebra $N_{6,2}$ we have $a_{1}=0$. The third basis element of $\operatorname{inn}(\mathbf{L})$ is either $b_{3}=x_{2}+c_{1} n_{3}+c_{2} n_{4}$ or $b_{3, k}=x_{1}+k x_{2}+c_{1} n_{3}+c_{2} n_{4}, k, c_{1}, c_{2} \in \mathbb{R}$. Only the subspace $\left\langle b_{1}, b_{2}, b_{3, k}\right\rangle$ is a 3 -dimensional Lie algebra only in the Lie algebras $N_{6, j}$, $j=3,6,11$. Then the Lie subalgebra $\operatorname{inn}(\mathbf{L})$ has the form: $\operatorname{inn}(\mathbf{L})_{a, a_{4}}=\left\langle n_{2}+a(1+\right.$ $\left.\beta) n_{4}, n_{1}+a n_{3}+a_{4} n_{4}, x_{1}+x_{2}\right\rangle$, where $a \neq 0, a_{4} \in \mathbb{R}, \beta \neq-1$ for $N_{6,6}$ and $\beta=0$ for $N_{6, j}, j=3,11$. Using the automorphism $\alpha\left(n_{i}\right)=a n_{i}, \alpha\left(x_{i}\right)=x_{i}, i=1,2$, $\alpha\left(n_{4}\right)=n_{4}, \alpha\left(n_{3}\right)=n_{3}-\frac{a_{4}}{a} n_{4}$ of the Lie algebras $N_{6, j}, j=3,6,11$, we can change the Lie algebra $\operatorname{inn}(\mathbf{L})_{a, a_{4}}$ onto $\operatorname{inn}(\mathbf{L})_{\beta}=\left\langle n_{2}+(1+\beta) n_{4}, n_{1}+n_{3}, x_{1}+x_{2}\right\rangle$ in the case $N_{6,6}^{\beta \neq-1}$ and $\beta=0$ for the Lie algebras $N_{6, j}, j=3,11$. Linear representations of the simply connected Lie groups $G_{j}$ of $N_{6, j}, j=3,6,11$, are given by:

for $N_{6,3}^{\alpha=0}$ :

$g\left(x_{1}, x_{2}, x_{3}, x_{4}, x_{5}, x_{6}\right) g\left(y_{1}, y_{2}, y_{3}, y_{4}, y_{5}, y_{6}\right)$

$$
=g\left(x_{1}+y_{1} e^{x_{6}}, x_{2}+y_{2} e^{x_{6}}+x_{5} y_{1} e^{x_{6}}, x_{3}+y_{3} e^{x_{5}},\right.
$$

for $N_{6,11}^{\alpha=0}$ :

$$
\left.x_{4}+y_{4} e^{x_{5}}+y_{3} x_{6} e^{x_{5}}, x_{5}+y_{5}, x_{6}+y_{6}\right) \text {, }
$$

$g\left(x_{1}+y_{1} e^{x_{6}}, x_{2}+y_{2} e^{x_{6}}+x_{5} y_{1} e^{x_{6}}, x_{3}+y_{3} e^{x_{5}}, x_{4}+y_{4} e^{x_{5}}+y_{3} x_{5} e^{x_{5}}, x_{5}+y_{5}, x_{6}+y_{6}\right)$, for $N_{6,6}^{\alpha=0, \beta \neq-1}$ :

$$
\begin{aligned}
g\left(x_{1}+y_{1} e^{x_{6}}, x_{2}+\right. & y_{2} e^{x_{6}}+x_{6} y_{1} e^{x_{6}}, \\
& \left.x_{3}+y_{3} e^{x_{5}}, x_{4}+y_{4} e^{x_{5}}+y_{3}\left(x_{5}+\beta x_{6}\right) e^{x_{5}}, x_{5}+y_{5}, x_{6}+y_{6}\right) .
\end{aligned}
$$

One has $\operatorname{Inn}(L)=\left\{g\left(u_{1}, u_{2}, u_{1},(1+\beta) u_{2}, s, s\right) ; u_{i}, s \in \mathbb{R}\right\}, i=1,2$, for $N_{6,6}^{\beta \neq-1}$ and $\beta=0$ in the cases $N_{6, j}, j=3,11$. Two arbitrary left transversals to the group $\operatorname{Inn}(L)$ in $G_{j}, j=3,6,11$, are

$$
\begin{aligned}
& A=\left\{g\left(f_{1}(k, l, m), f_{2}(k, l, m), k, l, m, f_{3}(k, l, m)\right), k, l, m \in \mathbb{R}\right\}, \\
& B=\left\{g\left(h_{1}(u, v, w), h_{2}(u, v, w), u, v, w, h_{3}(u, v, w)\right), u, v, w \in \mathbb{R}\right\},
\end{aligned}
$$

where $f_{i}(k, l, m): \mathbb{R}^{3} \rightarrow \mathbb{R}$ and $h_{i}(u, v, w): \mathbb{R}^{3} \rightarrow \mathbb{R}, i=1,2,3$, are continuous functions with $f_{i}(0,0,0)=h_{i}(0,0,0)=0$. For all $a \in A, b \in B$ the condition $a^{-1} b^{-1} a b \in \operatorname{Inn}(L)$ holds if and only if in all three cases the equation

$$
\begin{aligned}
e^{-h_{3}(u, v, w)} h_{1}(u, v, w)\left(1-e^{-f_{3}(k, l, m)}\right) & -e^{-f_{3}(k, l, m)} f_{1}(k, l, m)\left(1-e^{-h_{3}(u, v, w)}\right) \\
& =u e^{-w}\left(1-e^{-m}\right)-k e^{-m}\left(1-e^{-w}\right),
\end{aligned}
$$

and for $N_{6,3}^{\alpha=0}$,

$$
\begin{aligned}
e^{-h_{3}(u, v, w)}\left(1-e^{-f_{3}(k, l, m)}\right)\left(h_{2}(u, v, w)-w h_{1}(u, v, w)\right) \\
\quad-e^{-f_{3}(k, l, m)}\left(1-e^{-h_{3}(u, v, w)}\right)\left(f_{2}(k, l, m)-m f_{1}(k, l, m)\right) \\
\quad+e^{-f_{3}(k, l, m)-h_{3}(u, v, w)}\left(m h_{1}(u, v, w)-w f_{1}(k, l, m)\right) \\
=e^{-w}\left(1-e^{-m}\right)\left(v-h_{3}(u, v, w) u\right)-e^{-m}\left(1-e^{-w}\right)\left(l-f_{3}(k, l, m) k\right) \\
\quad+e^{-m-w}\left(f_{3}(k, l, m) u-h_{3}(u, v, w) k\right),
\end{aligned}
$$


for $N_{6,11}^{\alpha=0}$

$$
\begin{aligned}
& e^{-h_{3}(u, v, w)}\left(1-e^{-f_{3}(k, l, m)}\right)\left(h_{2}(u, v, w)-w h_{1}(u, v, w)\right) \\
& \quad-e^{-f_{3}(k, l, m)}\left(1-e^{-h_{3}(u, v, w)}\right)\left(f_{2}(k, l, m)-m f_{1}(k, l, m)\right) \\
& \quad+e^{-f_{3}(k, l, m)-h_{3}(u, v, w)}\left(m h_{1}(u, v, w)-w f_{1}(k, l, m)\right) \\
& =e^{-w}\left(1-e^{-m}\right)(v-w u)-e^{-m}\left(1-e^{-w}\right)(l-m k)+e^{-m-w}(m u-w k),
\end{aligned}
$$

for $N_{6,6}^{\alpha=0, \beta \neq-1}$,

$$
\begin{aligned}
(1+\beta) & {\left[e^{-h_{3}(u, v, w)}\left(1-e^{-f_{3}(k, l, m)}\right)\left(h_{2}(u, v, w)-h_{3}(u, v, w) h_{1}(u, v, w)\right)\right.} \\
& -e^{-f_{3}(k, l, m)}\left(1-e^{-h_{3}(u, v, w)}\right)\left(f_{2}(k, l, m)-f_{1}(k, l, m) f_{3}(k, l, m)\right) \\
& \left.+e^{-f_{3}(k, l, m)-h_{3}(u, v, w)}\left(f_{3}(k, l, m) h_{1}(u, v, w)-h_{3}(u, v, w) f_{1}(k, l, m)\right)\right] \\
= & e^{-w}\left(1-e^{-m}\right)\left[v-u\left(w+\beta h_{3}(u, v, w)\right)\right] \\
& -e^{-m}\left(1-e^{-w}\right)\left[l-k\left(m+\beta f_{3}(k, l, m)\right)\right] \\
& +e^{-m-w}\left[m u-w k+\beta\left(f_{3}(k, l, m) u-h_{3}(u, v, w) k\right)\right]
\end{aligned}
$$

are satisfied for all $u, v, w, k, l, m \in \mathbb{R}$. Equation (1) is satisfied precisely if one has $h_{3}(u, v, w)=w, h_{1}(u, v, w)=u, f_{1}(k, l, m)=k, f_{3}(k, l, m)=m$. Putting this into equations (2), (3), (4) we obtain in case (4)

$$
e^{-w}\left(1-e^{-m}\right)\left(v-(1+\beta) h_{2}(u, v, w)\right)=e^{-m}\left(1-e^{-w}\right)\left(l-(1+\beta) f_{2}(k, l, m)\right)
$$

and in cases (2), (3) equation (5) with $\beta=0$. Equation (5) holds if and only if one has $h_{2}(u, v, w)=v /(1+\beta), f_{2}(k, l, m)=l /(1+\beta)$, where $\beta=0$ in the cases $N_{6, j}^{\alpha=0}, j=3,11$, and $\beta \in \mathbb{R} \backslash\{-1\}$ in the case $N_{6,6}^{\alpha=0, \beta \neq-1}$. In all these cases $A \cup B$ does not generate the group $G_{j}, j=3,6,11$. By Lemma 4 the Lie algebras $N_{6, j}$, $j=3,6,11$, are not the Lie algebras of groups $\operatorname{Mult}(L)$ of 3 -dimensional topological loops $L$.

The Lie algebras $N_{6, j}, j \in\{29,31,32,33,34,38\}$, have non-abelian nilradical, and neither a subalgebra nor a factor Lie algebra of $N_{6, j}$ are isomorphic to an elementary filiform Lie algebra $\mathbf{f}_{n}, n \geq 4$. The Lie algebras $N_{6,31}$ and $N_{6,32}^{\alpha}$ have the ideal $\mathbf{i}=\left\langle n_{1}\right\rangle$. Both Lie algebras contain the nilpotent ideals: $\mathbf{s}_{1}=\left\langle n_{1}, n_{3}\right\rangle$, $\mathbf{s}_{2}=\left\langle n_{1}, n_{4}\right\rangle, \mathbf{s}_{3}=\left\langle n_{1}, n_{2}\right\rangle, \mathbf{s}_{4}=\left\langle n_{1}, n_{2}, n_{3}\right\rangle, \mathbf{s}_{5}=\left\langle n_{1}, n_{2}, n_{4}\right\rangle, \mathbf{s}_{6}=\left\langle n_{1}, n_{3}, n_{4}\right\rangle$, $\mathbf{n}_{\text {rad }}$. If $N_{6, j}, j=31,32$, would be the Lie algebra of the multiplication group of a 3-dimensional topological loop, then by Theorem 11 and Proposition 13a)(ii) there exist 2-dimensional ideals s of $N_{6, j}, j=31,32$, containing $\mathbf{i}$ such that the factor Lie algebras $N_{6, j} / \mathbf{s}, j=31,32$, are isomorphic to $\mathbf{l}_{2} \oplus \mathbf{l}_{2}$. The factor Lie algebra $N_{6,31} / \mathbf{s}_{1}$ is isomorphic to $\mathbf{l}_{\mathbf{2}} \oplus \mathbf{l}_{\mathbf{2}}$, whereas the factor Lie algebras $N_{6,31} / \mathbf{s}_{\mathbf{i}}$, $i=2,3$, are not so. The factor Lie algebra $N_{6,32}^{\alpha} / \mathbf{s}_{1}$ is isomorphic to $\mathbf{l}_{\mathbf{2}} \oplus \mathbf{l}_{\mathbf{2}}$ if and only if $\alpha=0$, but the factor Lie algebras $N_{6,32}^{\alpha=0} / \mathbf{s}_{\mathbf{i}}, i=2,3$, are not so. The factor Lie algebra $N_{6,32}^{\alpha} / \mathbf{s}_{3}$ is isomorphic to $\mathbf{l}_{\mathbf{2}} \oplus \mathbf{l}_{\mathbf{2}}$ precisely if $\alpha=1$, whereas the factor Lie algebras $N_{6,32}^{\alpha=1} / \mathbf{s}_{\mathbf{i}}, i=1,2$, are not so. Let $S_{k}$, respectively $N$ be the simply connected Lie groups of $\mathbf{s}_{\mathbf{k}}, k=1,2, \ldots, 6$, respectively $\mathbf{n}_{\text {rad }}$. For $N_{6,31}$, 
$N_{6,32}^{\alpha=0}$ the orbits $S_{i}(e), i=2,3, \ldots, 6$, and $N(e)$ are the same normal subgroup $M \cong \mathbb{R}^{2}$ of $L$ and for $N_{6,32}^{\alpha=1}$ we have $S_{j}(e)=N(e):=M, j=1,2,4,5,6$ (cf. Proposition $13 \mathrm{~b}$ ). The subgroup $M$ contains the normal subgroup $I(e) \cong \mathbb{R}$, where $I$ is the simply connected Lie group of $\mathbf{i}$, of $L$. For $m=4,5,6$ the intersections $\mathbf{s}_{m} \cap \mathbf{i n n}(\mathbf{L})$ have dimension 1 and $\mathbf{n}_{\text {rad }} \cap \mathbf{i n n}(\mathbf{L})$ has dimension 2 (see Proposition 13 b). Since for $N_{6,31}$ and $N_{6,32}^{\alpha=0}$ one has $\mathbf{s}_{i} \cap \operatorname{inn}(\mathbf{L})=\{0\}, i=2,3$ and for $N_{6,32}^{\alpha=1}$ we have $\mathbf{s}_{j} \cap \operatorname{inn}(\mathbf{L})=\{0\}, j=1,2$, the Lie algebra $\operatorname{inn}(\mathbf{L})$ contains the elements $b_{1}=n_{1}+a_{1} n_{3}, b_{2}=n_{2}+a_{2} n_{1}+a_{3} n_{4}, a_{1} a_{3} \neq 0$, in the cases $N_{6,31}$ and $N_{6,32}^{\alpha=0}$ and the elements $b_{1}=n_{1}+a_{1} n_{2}, b_{2}=n_{3}+a_{2} n_{1}+a_{3} n_{4}, a_{1} a_{3} \neq 0$ in the case $N_{6,32}^{\alpha=1}$. As $\left[b_{1}, b_{2}\right]=a_{1} n_{1}, a_{1} \neq 0$ in both cases $\operatorname{inn}(\mathbf{L})$ would contain the ideal $\left\langle n_{1}\right\rangle$ of $N_{6, j}, j=31,32$. This contradicts Lemma 5 .

The Lie algebras $N_{6,33}, N_{6,38}, N_{6,34}^{\alpha}$ and $N_{6,29}^{\alpha, \beta}$ have the ideals $\mathbf{i}_{1}=\left\langle n_{1}\right\rangle, \mathbf{i}_{2}=$ $\left\langle n_{4}\right\rangle$. The Lie algebras $N_{6, i}, i=29,38$, have the nilpotent ideals $\mathbf{s}_{1}=\left\langle n_{1}, n_{2}\right\rangle$, $\mathbf{s}_{2}=\left\langle n_{1}, n_{4}\right\rangle, \mathbf{s}_{3}=\left\langle n_{1}, n_{3}\right\rangle, \mathbf{s}_{4}=\left\langle n_{1}, n_{2}, n_{4}\right\rangle, \mathbf{s}_{5}=\left\langle n_{1}, n_{3}, n_{4}\right\rangle, \mathbf{s}_{6}=\left\langle n_{1}, n_{2}, n_{3}\right\rangle$, $\mathbf{n}_{\text {rad }}$ and the nilpotent ideals of $N_{6, j}, j=33,34$, are $\mathbf{s}_{1}, \mathbf{s}_{2}, \mathbf{s}_{4}, \mathbf{s}_{5}, \mathbf{n}_{\text {rad }}$. Denote by $I_{k}, S_{i}$ and $N$ the simply connected Lie groups of the ideals $\mathbf{i}_{k}, k=1,2, \mathbf{s}_{i}$, $i=1,2, \ldots, 6$ and $\mathbf{n}_{\text {rad }}$. The factor Lie algebras $N_{6, k} / \mathbf{s}_{2}, k \in\{29,33,38\}$, are isomorphic to $\mathbf{l}_{\mathbf{2}} \oplus \mathbf{l}_{\mathbf{2}}$ and $N_{6,34} / \mathbf{s}_{2}$ is isomorphic to $\mathbf{l}_{\mathbf{2}} \oplus \mathbf{l}_{\mathbf{2}}$ precisely if $\alpha=0$. If $N_{6, j}, j=29,33,34,38$, would be the Lie algebra of the multiplication group of a 3 dimensional topological loop, then the orbits $I_{k}(e), k=1,2$, are normal subgroups of $L$ isomorphic to $\mathbb{R}$ and the factor loops $L / I_{k}(e), k=1,2$, are isomorphic to $\mathcal{L}_{2}$ since the nilradical of $N_{6, j}$ are not abelian (cf. Proposition 13a)(i),(ii).

For $j=33,34$, the factor Lie algebras $N_{6, j} / \mathbf{s}_{1}$ are not isomorphic to $\mathbf{l}_{\mathbf{2}} \oplus \mathbf{l}_{\mathbf{2}}$. By Proposition $13 \mathrm{~b}$ ) the orbits $S_{l}(e), l=1,4,5$, and $N(e)$ are the same normal subgroup $M \cong \mathbb{R}^{2}$ of $L$ such that $S_{1} \cap \operatorname{Inn}(L)=\{1\}$, the intersections $S_{l} \cap \operatorname{Inn}(L)$ have dimension $1, l=4,5$, and $\operatorname{dim}(N \cap \operatorname{Inn}(L))=2$. For $N_{6,29}$ the factor Lie algebra $N_{29} / \mathbf{s}_{1}$ is isomorphic to $\mathbf{l}_{\mathbf{2}} \oplus \mathbf{l}_{\mathbf{2}}$ precisely if $\beta=0$ and $N_{29} / \mathbf{s}_{3}$ is isomorphic to $\mathbf{l}_{\mathbf{2}} \oplus \mathbf{l}_{\mathbf{2}}$ if and only if $\alpha=0$. If $\alpha \neq 0$, respectively $\beta \neq 0$ the orbits $S_{l}(e)$, $l=3,4,5,6$, and $N(e)$, respectively the orbits $S_{k}(e), k=1,4,5,6$, and $N(e)$ are the normal subgroup $M \cong \mathbb{R}^{2}$ of $L$. For $\alpha \neq 0$ one has $S_{3} \cap \operatorname{Inn}(L)=\{1\}$, whereas for $\beta \neq 0$ we have $S_{1} \cap \operatorname{Inn}(L)=\{1\}$, for $l=4,5,6$ the intersections $S_{l} \cap \operatorname{Inn}(L)$ have dimension 1 and $N \cap \operatorname{Inn}(L)$ has dimension 2 (cf. Proposition 13b). Since the factor Lie algebras $N_{6,38} / \mathbf{s}_{k}, k=1,3$, are not isomorphic to $\mathbf{l}_{\mathbf{2}} \oplus \mathbf{l}_{\mathbf{2}}$ the orbits $S_{l}(e), l=1,3,4,5,6$, and $N(e)$ are the same normal subgroup $M \cong \mathbb{R}^{2}$ of $L$ and for $l=1,3$, one has $S_{l} \cap \operatorname{Inn}(L)=\{1\}$, for $l=4,5,6$, the intersections $S_{l} \cap \operatorname{Inn}(L)$ have dimension 1 , and $\operatorname{dim}(N \cap \operatorname{Inn}(L))=2$ (cf. Proposition 13b). In all cases the normal subgroup $I_{k}(e), k=1,2$, are in $M$. For $j=29,33,34,38$, the Lie algebra $\operatorname{inn}(\mathbf{L})$ lies in one of the following ideals: $\mathbf{v}_{1}=\left\langle n_{1}, n_{2}, n_{3}, n_{4}, x_{1}\right\rangle, \mathbf{v}_{2, k}=$ $\left\langle n_{1}, n_{2}, n_{3}, n_{4}, x_{2}+k x_{1}\right\rangle, k \in \mathbb{R}$. If for $N_{6, j}, j=33,34$, the Lie algebra $\operatorname{inn}(\mathbf{L})$ would contain the basis elements $b_{1}=n_{2}+a_{1} n_{4}+a_{2} n_{1}, b_{2}=n_{3}+a_{3} n_{4}+a_{4} n_{1}$, and for $N_{6,29}$ either the basis elements $b_{1}=n_{1}+a_{1} n_{2}, b_{2}=n_{3}+a_{2} n_{4}+a_{3} n_{1}$, or the basis elements $b_{1}=n_{1}+a_{1} n_{3}, b_{2}=n_{2}+a_{2} n_{4}+a_{3} n_{1}$ with $a_{1} \neq 0$ would be in $\operatorname{inn}(\mathbf{L})$, then $\operatorname{inn}(\mathbf{L})$ would contain the ideal $\left\langle n_{1}\right\rangle$ of $N_{6, j}, j=29,33,34$, since one has $\left[b_{1}, b_{2}\right]=c n_{1}, c \neq 0$. This is a contradiction to Lemma 5 . Otherwise for $N_{6, j}, j=29,33,34,38$, the Lie algebra $\operatorname{inn}(\mathbf{L})$ would contain the basis elements either $b_{1}^{\prime}=n_{1}+a_{1} n_{4}, b_{2}^{\prime}=n_{2}+a_{2} n_{3}+a_{3} n_{4}, b_{3}^{\prime}=x_{1}+c_{1} n_{3}+c_{2} n_{4}$ or $b_{1}^{\prime}, b_{2}^{\prime}$, 
$b_{3, k}^{\prime}=x_{2}+k x_{1}+c_{1} n_{3}+c_{2} n_{4}$, where $a_{1} a_{2} \neq 0, k, c_{1}, c_{2}, a_{3} \in \mathbb{R}$. The subspaces $\left\langle b_{1}^{\prime}, b_{2}^{\prime}, b_{3}^{\prime}\right\rangle,\left\langle b_{1}^{\prime}, b_{2}^{\prime}, b_{3, k}^{\prime}\right\rangle$ are not 3 -dimensional Lie algebras. This proves that none of the Lie algebras $N_{6, j}, j=29,31,32,33,34,38$, are the Lie algebras of the group $\operatorname{Mult}(L)$ of a 3 -dimensional topological loop $L$.

\section{Three-dimensional topological loops corresponding to six-dimensional solvable Lie algebras with four-dimensional abelian nilradical and one-dimensional centre}

Theorem 15. If $L$ is a connected topological proper loop of dimension 3 such that the Lie algebra of its multiplication group $\operatorname{Mult}(L)$ is a 6-dimensional solvable indecomposable Lie algebra having 4-dimensional nilradical, then $L$ is centrally nilpotent of class 2 .

Proof. By Lemma 6 we may assume that $L$ is homoemorphic to $\mathbb{R}^{3}$. By Proposition 14 it remains to deal with the 6-dimensional solvable indecomposable Lie algebras $N_{6, i}, i=20, \ldots, 27$, with abelian nilradical and 1-dimensional centre (cf. [T, Table II, p. 1348]). By Theorem 12(a) we have to prove that there is a normal subgroup $N \cong \mathbb{R}$ of $L$ such that the factor loop $L / N$ is isomorphic to $\mathbb{R}^{2}$. The Lie algebra $N_{6,20}^{a, b}, a^{2}+b^{2} \neq 0$, has the ideals $\mathbf{i}_{1}=\left\langle n_{1}\right\rangle, \mathbf{i}_{2}=\left\langle n_{2}\right\rangle, \mathbf{i}_{3}=\left\langle n_{3}\right\rangle$, $\mathbf{i}_{4}=\left\langle n_{4}\right\rangle$. If $N_{6,20}^{a, b}$ is the Lie algebra of the multiplication group of a 3 -dimensional connected topological loop $L$, then the orbits $I_{k}(e), k \in\{1,2,3,4\}$, are normal subgroups of $L$ isomorphic to $\mathbb{R}$ (cf. Lemma 3 ). The Lie algebra $N_{6,20}^{a, b}$ has no factor Lie algebra isomorphic to an elementary filiform Lie algebra. Hence the factor loops $L / I_{k}(e), k \in\{1,2,3,4\}$, are isomorphic either to $\mathcal{L}_{2}$ or to $\mathbb{R}^{2}$ (cf. Proposition 13(i),(ii)). If all factor loops $L / I_{k}(e), k \in\{1,2,3,4\}$, are isomorphic to $\mathcal{L}_{2}$, then by Proposition 13 (ii) there are 2-dimensional ideals $\mathbf{s}_{k}, k \in\{1,2,3,4\}$ such that $\mathbf{i}_{k} \subset \mathbf{s}_{k}$ and the factor Lie algebras $N_{6,20}^{a, b} / \mathbf{s}_{k}$ are isomorphic to $\mathbf{l}_{2} \oplus \mathbf{l}_{2}$. For the ideal $\mathbf{s}_{1}=\mathbf{s}_{2}=\left\langle n_{1}, n_{2}\right\rangle$ one has $N_{6,20}^{a, b} / \mathbf{s}_{k} \cong \mathbf{l}_{2} \oplus \mathbf{l}_{2}, k=1,2$. The factor Lie algebra $N_{6,20}^{a, b} /\left\langle n_{1}, n_{3}\right\rangle$ is isomorphic to $\mathbf{l}_{2} \oplus \mathbf{l}_{2}$ if and only if $a=0$ and $N_{6,20}^{a, b} /\left\langle n_{1}, n_{4}\right\rangle$ is isomorphic to $\mathbf{l}_{2} \oplus \mathbf{l}_{2}$ precisely if $b=0$. This contradiction to $a^{2}+b^{2} \neq 0$ yields that at least one of the factor loops $L / I_{k}(e), k \in\{1,2,3,4\}$, is isomorphic to $\mathbb{R}^{2}$. For such $k \in\{1,2,3,4\}$ the orbit $I_{k}(e)$ is the requested normal subgroup of $L$ in Theorem $12(\mathrm{a})$.

The Lie algebras $N_{6,21}^{a}, N_{6,22}^{\varepsilon, a}, N_{6,24}, N_{6,25}^{a, b}, N_{6,26}^{a}, N_{6,27}^{\varepsilon}$ have the ideal $\mathbf{i}=\left\langle n_{2}\right\rangle$ and the unique 1-dimensional ideal of the Lie algebra $N_{6,23}^{a, \varepsilon}$ is its centre $\mathbf{i}=\left\langle n_{4}\right\rangle$. There does not exist any ideal $\mathbf{s}$ of these Lie algebras $N_{6, i}$ containing $\mathbf{i}$ such that the factor Lie algebras $N_{6, i} / \mathbf{s}$ are isomorphic either to $\mathbf{l}_{2} \oplus \mathbf{l}_{2}$ or to $\mathbf{f}_{4}$. If $N_{6, i}, i=$ $21, \ldots, 27$, would be the Lie algebra of the multiplication group of a 3 -dimensional connected topological loop $L$, then the factor loop $L / I(e)$ is isomorphic to $\mathbb{R}^{2}$ (cf. Proposition 13(i)). Hence the orbit $I(e)$ satisfies the assertion of Theorem 12(a).

Proposition 16. Let $\mathbf{g}$ be a 6-dimensional solvable indecomposable Lie algebra having 4-dimensional abelian nilradical $\mathbf{n}_{\mathrm{rad}}$ and 1-dimensional centre. Let $\mathbf{k}$ be a 3-dimensional abelian subalgebra of $\mathbf{g}$ which does not contain any non-zero ideal 
of $\mathbf{g}$ and the normalizer $N_{\mathbf{g}}(\mathbf{k})$ of $\mathbf{k}$ in $\mathbf{g}$ is $\mathbf{n}_{\mathrm{rad}}$. Then for the Lie algebra $\mathbf{g}$ and up to automorphisms of $\mathbf{g}$ for the subalgebra $\mathbf{k}$ we have one of the following cases:

(a) $\mathbf{g}_{1}:=N_{6,20}^{a, b}, \mathbf{k}_{1}=\left\langle n_{2}+n_{1}, n_{3}+n_{1}, n_{4}+n_{1}\right\rangle$.

(b) $\mathbf{g}_{2}:=N_{6,21}^{a}, \mathbf{g}_{3}:=N_{6,24}, \mathbf{k}_{2}=\mathbf{k}_{3}=\left\langle n_{2}+n_{1}, n_{3}+\varepsilon_{1} n_{1}, n_{4}+n_{1}\right\rangle, \varepsilon_{1}=0,1$.

(c) $\mathbf{g}_{4}:=N_{6,25}^{a, b}, \mathbf{g}_{5}:=N_{6,26}^{a}, \mathbf{k}_{4}=\mathbf{k}_{5}=\left\langle n_{2}+n_{1}, n_{3}+\varepsilon_{1} n_{1}, n_{4}+\varepsilon_{2} n_{1}\right\rangle, \varepsilon_{i}=0,1$, $i=1,2$, at least one of $\left\{\varepsilon_{1}, \varepsilon_{2}\right\}$ is different from 0 .

(d) $\mathbf{g}_{6}:=N_{6,27}^{\varepsilon}, \mathbf{k}_{6}=\left\langle n_{1}+\varepsilon_{1} n_{2}, n_{3}+\varepsilon_{2} n_{2}, n_{4}+\varepsilon_{3} n_{2}\right\rangle, \varepsilon_{i}=0,1, i=1,2,3$, such that at least one of $\left\{\varepsilon_{2}, \varepsilon_{3}\right\}$ is different from 0 .

(e) $\mathbf{g}_{7}:=N_{6,23}^{a, \varepsilon}, \mathbf{k}_{7}=\left\langle n_{1}+\varepsilon_{1} n_{4}, n_{2}+\varepsilon_{2} n_{4}, n_{3}+\varepsilon_{3} n_{4}\right\rangle, \varepsilon_{i}=0,1, i=1,2,3$, such that at least one of $\left\{\varepsilon_{1}, \varepsilon_{2}\right\}$ is different from 0 .

(f) $\mathbf{g}_{8}:=N_{6,22}^{a, \varepsilon}, \mathbf{k}_{3}=\left\langle n_{1}+n_{4}, n_{2}+n_{4}, n_{3}+\varepsilon_{1} n_{4}\right\rangle, \varepsilon_{1}=0,1$.

Proof. The 6-dimensional indecomposable solvable Lie algebras with abelian nilradical and 1-dimensional centre in [T, Table II, p. 1348] are the Lie algebras $\mathbf{g}_{i}$, $i=1, \ldots, 8$. The Lie algebras $\mathbf{g}_{i}, i=1, \ldots, 5$, have the centre $\mathbf{z}=\left\langle n_{1}\right\rangle$. For these Lie algebras the subalgebra $\mathbf{k}$ has the form

$$
\mathbf{k}_{a_{2}, a_{3}, a_{4}}=\left\langle n_{2}+a_{2} n_{1}, n_{3}+a_{3} n_{1}, n_{4}+a_{4} n_{1}\right\rangle,
$$

such that

in the case $\mathbf{g}_{1}: a_{2} a_{3} a_{4} \neq 0$, since $\left\langle n_{2}\right\rangle,\left\langle n_{3}\right\rangle,\left\langle n_{4}\right\rangle$ are ideals of $\mathbf{g}_{1}$,

in the cases $\mathbf{g}_{2}, \mathbf{g}_{3}: a_{2} a_{4} \neq 0$ because $\left\langle n_{4}\right\rangle$ and $\left\langle n_{2}\right\rangle$ are ideals of $\mathbf{g}_{i}, i=2,3$,

in the cases $\mathbf{g}_{4}, \mathbf{g}_{5}: a_{2} \neq 0$ and at least one of the constants $\left\{a_{3}, a_{4}\right\}$ is different from 0 since $\left\langle n_{2}\right\rangle$ and $\left\langle n_{3}, n_{4}\right\rangle$ are ideals of $\mathbf{g}_{i}, i=4,5$.

For the Lie algebras $\mathbf{g}_{i}, i=1, \ldots, 5$, using the automorphism $\alpha\left(n_{1}\right)=n_{1}$, $\alpha\left(x_{i}\right)=x_{i}, i=1,2, \alpha\left(n_{2}\right)=a_{2} n_{2}, \alpha\left(n_{i}\right)=a_{i} n_{i}, i=3,4$, if $a_{i} \neq 0$, otherwise $\alpha\left(n_{i}\right)=n_{i}$, we can change $\mathbf{k}_{a_{2}, a_{3}, a_{4}}$ onto $\mathbf{k}=\left\langle n_{2}+n_{1}, n_{3}+\varepsilon_{1} n_{1}, n_{4}+\varepsilon_{2} n_{1}\right\rangle$, such that $\varepsilon_{1}$, respectively $\varepsilon_{2}$ is equal to 0 or 1 , according whether $a_{3}$, respectively $a_{4}$ is 0 or $\neq 0$. The Lie algebra $\mathbf{g}_{6}$ has the centre $\mathbf{z}=\left\langle n_{2}\right\rangle$ and hence for the subalgebra $\mathbf{k}$ one has $\mathbf{k}_{a_{1}, a_{3}, a_{4}}=\left\langle n_{1}+a_{1} n_{2}, n_{3}+a_{3} n_{2}, n_{4}+a_{4} n_{2}\right\rangle$, such that $a_{3} \neq 0$ or $a_{4} \neq 0$ because $\left\langle n_{3}, n_{4}\right\rangle$ is an ideal of $\mathbf{g}_{6}$. Using the automorphism $\alpha\left(n_{2}\right)=n_{2}, \alpha\left(x_{i}\right)=x_{i}$, $i=1,2, \alpha\left(n_{i}\right)=a_{i} n_{i}$, if $a_{i} \neq 0$, otherwise $\alpha\left(n_{i}\right)=n_{i}, i=1,3,4$, we can reduce the Lie algebra $\mathbf{k}_{a_{1}, a_{3}, a_{4}}$ to $\mathbf{k}=\left\langle n_{1}+\varepsilon_{1} n_{2}, n_{3}+\varepsilon_{2} n_{2}, n_{4}+\varepsilon_{3} n_{2}\right\rangle, \varepsilon_{i}=0,1, i=1,2,3$, such that at least one of $\left\{\varepsilon_{2}, \varepsilon_{3}\right\}$ is different from 0 . The centre of the Lie algebras $\mathbf{g}_{i}, i=7,8$, is $\left\langle n_{4}\right\rangle$. For the subalgebra $\mathbf{k}$ of $\mathbf{g}_{i}, i=7,8$, we obtain

$$
\mathbf{k}_{a_{1}, a_{2}, a_{3}}=\left\langle n_{1}+a_{1} n_{4}, n_{2}+a_{2} n_{4}, n_{3}+a_{3} n_{4}\right\rangle,
$$

such that

in the case $\mathbf{g}_{7}: a_{1} \neq 0$ or $a_{2} \neq 0$, since $\left\langle n_{1}, n_{2}\right\rangle$ is an ideal of $\mathbf{g}_{7}$, in the case $\mathbf{g}_{8}: a_{1} a_{2} \neq 0$ because $\left\langle n_{1}\right\rangle$ and $\left\langle n_{2}\right\rangle$ are ideals of $\mathbf{g}_{8}$.

For $\mathbf{g}_{i}, i=7,8$, using the automorphism $\alpha\left(n_{4}\right)=n_{4}, \alpha\left(x_{i}\right)=x_{i}, i=1,2$, $\alpha\left(n_{i}\right)=a_{i} n_{i}$, if $a_{i} \neq 0$, otherwise $\alpha\left(n_{i}\right)=n_{i}, i=1,2,3$, we can change $\mathbf{k}_{a_{1}, a_{2}, a_{3}}$ onto $\mathbf{k}=\left\langle n_{1}+\varepsilon_{1} n_{4}, n_{2}+\varepsilon_{2} n_{4}, n_{3}+\varepsilon_{3} n_{4}\right\rangle$, such that $\varepsilon_{i}$ is equal to 0 or 1 , according whether $a_{i}=0$ or $a_{i} \neq 0, i=1,2,3$. 
Theorem 17. Let $L$ be a connected simply connected topological proper loop of dimension 3 such that the Lie algebra of its multiplication group $\operatorname{Mult}(L)$ is a 6 dimensional solvable indecomposable Lie algebra having 4-dimensional nilradical. Then the following Lie groups are the multiplication groups $\operatorname{Mult}(L)$ and the following subgroups are the inner mapping groups $\operatorname{Inn}(L)$ of $L$ :

1) $\operatorname{Mult}(L)_{1}$ is given by the multiplication

$$
\begin{aligned}
& g\left(x_{1}, x_{2}, x_{3}, x_{4}, x_{5}, x_{6}\right) g\left(y_{1}, y_{2}, y_{3}, y_{4}, y_{5}, y_{6}\right) \\
& =g\left(x_{1}+y_{1}, x_{2}+y_{2}+x_{5} y_{1}, x_{3}+y_{3} e^{x_{6}} \cos \left(x_{5}\right)-y_{4} e^{x_{6}} \sin \left(x_{5}\right),\right. \\
& \left.x_{4}+y_{4} e^{x_{6}} \cos \left(x_{5}\right)+y_{3} e^{x_{6}} \sin \left(x_{5}\right), x_{5}+y_{5}, x_{6}+y_{6}\right),
\end{aligned}
$$

$\operatorname{Inn}(L)_{1}$ is the subgroup $\left\{g\left(u_{1}, \varepsilon_{1} u_{1}+\varepsilon_{2} u_{2}+\varepsilon_{3} u_{3}, u_{2}, u_{3}, 0,0\right) ; u_{1}, u_{2}, u_{3} \in \mathbb{R}\right\}$, $\varepsilon_{k} \in\{0,1\}, k=1,2,3$, such that $\varepsilon_{2}^{2}+\varepsilon_{3}^{2} \neq 0$.

2) The multiplication of the group $\operatorname{Mult}(L)_{2}$ is defined by

$$
\begin{gathered}
g\left(x_{1}, x_{2}, x_{3}, x_{4}, x_{5}, x_{6}\right) g\left(y_{1}, y_{2}, y_{3}, y_{4}, y_{5}, y_{6}\right) \\
=g\left(x_{1}+y_{1} e^{x_{5}} \cos \left(x_{6}\right)-y_{2} e^{x_{5}} \sin \left(x_{6}\right), x_{2}+y_{2} e^{x_{5}} \cos \left(x_{6}\right)+y_{1} e^{x_{5}} \sin \left(x_{6}\right),\right. \\
\left.x_{3}+y_{3}, x_{4}+y_{4}+\left(a x_{6}+x_{5}\right) y_{3}, x_{5}+y_{5}, x_{6}+y_{6}\right), a \in \mathbb{R}
\end{gathered}
$$

$\operatorname{Inn}(L)_{2}$ is the subgroup $\left\{g\left(u_{1}, u_{2}, u_{3}, \varepsilon_{1} u_{1}+\varepsilon_{2} u_{2}+\varepsilon_{3} u_{3}, 0,0\right) ; u_{1}, u_{2}, u_{3} \in \mathbb{R}\right\}$, $\varepsilon_{k} \in\{0,1\}, k=1,2,3$, such that $\varepsilon_{1}^{2}+\varepsilon_{2}^{2} \neq 0$.

3) The multiplication of the group $\operatorname{Mult}(L)_{3}$ is given by

$$
\begin{aligned}
& g\left(x_{1}, x_{2}, x_{3}, x_{4}, x_{5}, x_{6}\right) g\left(y_{1}, y_{2}, y_{3}, y_{4}, y_{5}, y_{6}\right) \\
& =g\left(x_{1}+y_{1} e^{x_{5}+a x_{6}}, x_{2}+y_{2} e^{x_{6}}, x_{3}+y_{3},\right. \\
& \left.x_{4}+y_{4}+x_{5} y_{3}, x_{5}+y_{5}, x_{6}+y_{6}\right), a \in \mathbb{R} \backslash\{0\}
\end{aligned}
$$

and $\operatorname{Inn}(L)_{3}$ is $\left\{g\left(u_{1}, u_{2}, u_{3}, u_{1}+u_{2}+\varepsilon u_{3}, 0,0\right) ; u_{1}, u_{2}, u_{3} \in \mathbb{R}\right\}, \varepsilon=0,1$.

Proof. According to Theorem 15 the centre $Z(L)$ of the simply connected loop $L$ is isomorphic to $\mathbb{R}$ and the factor loop $L / Z(L)$ is isomorphic to $\mathbb{R}^{2}$. By Proposition $13(\mathrm{i})$ the Lie algebra $\mathbf{g}$ of the group $\operatorname{Mult}(L)$ of $L$ has abelian nilradical and $\mathbf{n}_{\text {rad }}=$ $\mathbf{z} \oplus \operatorname{inn}(\mathbf{L})$, where $\mathbf{z}$ is the centre of $\mathbf{g}$ and $\operatorname{inn}(\mathbf{L})$ is the Lie algebra of the group $\operatorname{Inn}(L)$. Hence $\operatorname{inn}(\mathbf{L})$ is a 3 -dimensional abelian subalgebra of $\mathbf{g}$ which does not contain any non-zero ideal of $\mathbf{g}$ and the normalizer $N_{\mathbf{g}}(\mathbf{i n n}(\mathbf{L}))$ coincides with $\mathbf{n}_{\text {rad }}$ (cf. Lemma 5). It follows from Proposition 16 that the pairs $\left(\mathbf{g}_{i}, \mathbf{k}_{i}\right), i=1, \ldots, 8$, can occur as the Lie algebras of the group $\operatorname{Mult}(L)$ and the $\operatorname{subgroup} \operatorname{Inn}(L)$. Linear representations of the simply connected Lie groups $G_{i}$ of $\mathbf{g}_{i}$ are given in this order by

$$
\begin{gathered}
i=1: g\left(x_{1}, x_{2}, x_{3}, x_{4}, x_{5}, x_{6}\right) g\left(y_{1}, y_{2}, y_{3}, y_{4}, y_{5}, y_{6}\right) \\
=g\left(x_{1}+y_{1}+x_{6} y_{5}, x_{2}+y_{2} e^{a x_{5}+b x_{6}}, x_{3}+y_{3} e^{x_{6}},\right. \\
\left.x_{4}+y_{4} e^{x_{5}}, x_{5}+y_{5}, x_{6}+y_{6}\right), \\
i=2: g\left(x_{1}, x_{2}, x_{3}, x_{4}, x_{5}, x_{6}\right) g\left(y_{1}, y_{2}, y_{3}, y_{4}, y_{5}, y_{6}\right) \\
= \\
g\left(x_{1}+y_{1}+x_{6} y_{5}, x_{2}+y_{2} e^{x_{5}+a x_{6}}, x_{3}+y_{3} e^{x_{6}},\right. \\
\left.x_{4}+y_{4} e^{x_{6}}+x_{3} y_{5}, x_{5}+y_{5}, x_{6}+y_{6}\right),
\end{gathered}
$$




$$
\begin{gathered}
i=3: g\left(x_{1}, x_{2}, x_{3}, x_{4}, x_{5}, x_{6}\right) g\left(y_{1}, y_{2}, y_{3}, y_{4}, y_{5}, y_{6}\right) \\
=g\left(x_{1}+y_{1}+x_{5} y_{6}, x_{2}+y_{2} e^{x_{6}}, x_{3}+y_{3} e^{x_{5}},\right. \\
\left.x_{4}+y_{4} e^{x_{5}}+x_{5} e^{x_{5}} y_{3}, x_{5}+y_{5}, x_{6}+y_{6}\right), \\
i=4: g\left(x_{1}, x_{2}, x_{3}, x_{4}, x_{5}, x_{6}\right) g\left(y_{1}, y_{2}, y_{3}, y_{4}, y_{5}, y_{6}\right) \\
=g\left(x_{1}+y_{1}-x_{6} y_{5}, x_{2}+y_{2} e^{a x_{5}+b x_{6}},\right. \\
y_{3}+x_{3} \cos \left(y_{5}\right) e^{y_{6}}-x_{4} \sin \left(y_{5}\right) e^{y_{6}}, \\
\left.y_{4}+x_{4} \cos \left(y_{5}\right) e^{y_{6}}+x_{3} \sin \left(y_{5}\right) e^{y_{6}}, x_{5}+y_{5}, x_{6}+y_{6}\right), \\
i=5: g\left(x_{1}, x_{2}, x_{3}, x_{4}, x_{5}, x_{6}\right) g\left(y_{1}, y_{2}, y_{3}, y_{4}, y_{5}, y_{6}\right) \\
=g\left(x_{1}+y_{1}+x_{6} y_{5}, x_{2}+y_{2} e^{x_{6}},\right. \\
x_{3}+y_{3} \cos \left(x_{5}\right) e^{a x_{5}}+y_{4} \sin \left(x_{5}\right) e^{a x_{5}}, \\
\left.x_{4}+y_{4} \cos \left(x_{5}\right) e^{a x_{5}}-y_{3} \sin \left(x_{5}\right) e^{a x_{5}}, x_{5}+y_{5}, x_{6}+y_{6}\right), \\
i=6: \varepsilon=0: g\left(x_{1}, x_{2}, x_{3}, x_{4}, x_{5}, x_{6}\right) g\left(y_{1}, y_{2}, y_{3}, y_{4}, y_{5}, y_{6}\right)
\end{gathered}
$$$$
=g\left(x_{1}+y_{1}, x_{2}+y_{2}+x_{5} y_{1}\right.
$$$$
x_{3}+y_{3} e^{x_{6}} \cos \left(x_{5}\right)-y_{4} e^{x_{6}} \sin \left(x_{5}\right) \text {, }
$$$$
\left.x_{4}+y_{4} e^{x_{6}} \cos \left(x_{5}\right)+y_{3} e^{x_{6}} \sin \left(x_{5}\right), x_{5}+y_{5}, x_{6}+y_{6}\right),
$$

$$
\begin{aligned}
i=6: \varepsilon=1: g( & \left.x_{1}, x_{2}, x_{3}, x_{4}, x_{5}, x_{6}\right) g\left(y_{1}, y_{2}, y_{3}, y_{4}, y_{5}, y_{6}\right) \\
= & g\left(x_{1}+y_{1}+x_{5} y_{6}, x_{2}+y_{2}+x_{5} y_{1}\right. \\
& +\frac{1}{2} x_{5}^{2} y_{6}, y_{3}+x_{3} e^{y_{6}} \cos \left(y_{5}\right)-x_{4} e^{y_{6}} \sin \left(y_{5}\right), \\
& \left.y_{4}+x_{4} e^{y_{6}} \cos \left(y_{5}\right)+x_{3} e^{y_{6}} \sin \left(y_{5}\right), x_{5}+y_{5}, x_{6}+y_{6}\right),
\end{aligned}
$$

$i=7: \varepsilon=0: g\left(x_{1}, x_{2}, x_{3}, x_{4}, x_{5}, x_{6}\right) g\left(y_{1}, y_{2}, y_{3}, y_{4}, y_{5}, y_{6}\right)$

$$
\begin{aligned}
= & g\left(x_{1}+y_{1} e^{x_{5}} \cos \left(x_{6}\right)-y_{2} e^{x_{5}} \sin \left(x_{6}\right),\right. \\
& x_{2}+y_{2} e^{x_{5}} \cos \left(x_{6}\right)+y_{1} e^{x_{5}} \sin \left(x_{6}\right), \\
& \left.x_{3}+y_{3}, x_{4}+y_{4}+\left(a x_{6}+x_{5}\right) y_{3}, x_{5}+y_{5}, x_{6}+y_{6}\right),
\end{aligned}
$$

$i=7: \varepsilon=1, a=0: g\left(x_{1}, x_{2}, x_{3}, x_{4}, x_{5}, x_{6}\right) g\left(y_{1}, y_{2}, y_{3}, y_{4}, y_{5}, y_{6}\right)$

$$
\begin{aligned}
= & g\left(x_{1}+y_{1} e^{x_{5}} \cos \left(x_{6}\right)+y_{2} e^{x_{5}} \sin \left(x_{6}\right),\right. \\
& x_{2}+y_{2} e^{x_{5}} \cos \left(x_{6}\right)-y_{1} e^{x_{5}} \sin \left(x_{6}\right), x_{3}+y_{3}+x_{5} y_{6}, \\
& \left.x_{4}+y_{4}+x_{5} y_{3}+\frac{1}{2} x_{5}^{2} y_{6}, x_{5}+y_{5}, x_{6}+y_{6}\right),
\end{aligned}
$$

$i=7: \varepsilon=1, a \neq 0: g\left(x_{1}, x_{2}, x_{3}, x_{4}, x_{5}, x_{6}\right) g\left(y_{1}, y_{2}, y_{3}, y_{4}, y_{5}, y_{6}\right)$

$$
\begin{aligned}
= & g\left(x_{1}+y_{1} e^{x_{5}} \cos \left(x_{6}\right)+y_{2} e^{x_{5}} \sin \left(x_{6}\right),\right. \\
& x_{2}+y_{2} e^{x_{5}} \cos \left(x_{6}\right)-y_{1} e^{x_{5}} \sin \left(x_{6}\right), \\
& x_{3}+y_{3}+\left(a x_{6}+x_{5}\right) y_{5}, \\
& x_{4}+y_{4}+\left(a x_{6}+x_{5}\right) y_{3}+\frac{1}{2}\left(a x_{6}+x_{5}\right)^{2} y_{5}, \\
& \left.x_{5}+y_{5}, x_{6}+y_{6}\right),
\end{aligned}
$$

$i=8: \varepsilon=0: g\left(x_{1}, x_{2}, x_{3}, x_{4}, x_{5}, x_{6}\right) g\left(y_{1}, y_{2}, y_{3}, y_{4}, y_{5}, y_{6}\right)$

$$
\begin{aligned}
= & g\left(x_{1}+y_{1} e^{x_{5}+a x_{6}}, x_{2}+y_{2} e^{x_{6}}, x_{3}+y_{3},\right. \\
& \left.x_{4}+y_{4}+x_{5} y_{3}, x_{5}+y_{5}, x_{6}+y_{6}\right),
\end{aligned}
$$

$i=8: \varepsilon=1: g\left(x_{1}, x_{2}, x_{3}, x_{4}, x_{5}, x_{6}\right) g\left(y_{1}, y_{2}, y_{3}, y_{4}, y_{5}, y_{6}\right)$

$$
\begin{aligned}
= & g\left(x_{1}+y_{1} e^{x_{5}+a x_{6}}, x_{2}+y_{2} e^{x_{6}}, x_{3}+y_{3}+x_{5} y_{6},\right. \\
& \left.x_{4}+y_{4}+x_{5} y_{3}+\frac{1}{2} x_{5}^{2} y_{6}, x_{5}+y_{5}, x_{6}+y_{6}\right),
\end{aligned}
$$


where $a, b \in \mathbb{R}$ such that for $i=1,4$ one has $a^{2}+b^{2} \neq 0$ and for $i=8, \varepsilon=0$ we have $a \neq 0$ (cf. [RT, pp. 16-21]). Using these linear representations the Lie groups of the Lie algebras $\mathbf{k}_{i}$ are:

for $i=1: \operatorname{Inn}(L)=\left\{g\left(u_{1}+u_{2}+u_{3}, u_{1}, u_{2}, u_{3}, 0,0\right) ; u_{j} \in \mathbb{R}\right\}, j=1,2,3$;

for $i=2,3: \operatorname{Inn}(L)=\left\{g\left(u_{1}+\varepsilon u_{2}+u_{3}, u_{1}, u_{2}, u_{3}, 0,0\right) ; u_{j} \in \mathbb{R}\right\}, j=1,2,3$, $\varepsilon=0,1$;

for $i=4,5: \operatorname{Inn}(L)=\left\{g\left(u_{1}+\varepsilon_{2} u_{2}+\varepsilon_{3} u_{3}, u_{1}, u_{2}, u_{3}, 0,0\right) ; u_{j} \in \mathbb{R}\right\}, j=1,2,3$, $\varepsilon_{k}=0,1, k=2,3$ such that at least one of $\left\{\varepsilon_{2}, \varepsilon_{3}\right\}$ is different from 0 ;

for $i=6: \operatorname{Inn}(L)=\left\{g\left(u_{1}, \varepsilon_{1} u_{1}+\varepsilon_{2} u_{2}+\varepsilon_{3} u_{3}, u_{2}, u_{3}, 0,0\right) ; u_{j} \in \mathbb{R}\right\}, j=1,2,3$, $\varepsilon_{k}=0,1, k=1,2,3$, such that at least one of $\left\{\varepsilon_{2}, \varepsilon_{3}\right\}$ is different from 0 ;

for $i=7: \operatorname{Inn}(L)=\left\{g\left(u_{1}, u_{2}, u_{3}, \varepsilon_{1} u_{1}+\varepsilon_{2} u_{2}+\varepsilon_{3} u_{3}, 0,0\right) ; u_{j} \in \mathbb{R}\right\}, j=1,2,3$, $\varepsilon_{k}=0,1, k=1,2,3$, such that at least one of $\left\{\varepsilon_{1}, \varepsilon_{2}\right\}$ is different from 0 ;

for $i=8: \operatorname{Inn}(L)=\left\{g\left(u_{1}, u_{2}, u_{3}, u_{1}+u_{2}+\varepsilon u_{3}, 0,0\right) ; u_{j} \in \mathbb{R}\right\}, j=1,2,3, \varepsilon=0,1$.

Two arbitrary left transversals to the group $\operatorname{Inn}(L)$ in $G_{i}, i=1, \ldots, 5$, are

$$
\begin{aligned}
& A=\left\{g\left(k, f_{1}(k, l, m), f_{2}(k, l, m), f_{3}(k, l, m), l, m\right), k, l, m \in \mathbb{R}\right\}, \\
& B=\left\{g\left(u, h_{1}(u, v, w), h_{2}(u, v, w), h_{3}(u, v, w), v, w\right), u, v, w \in \mathbb{R}\right\},
\end{aligned}
$$

those to the group $\operatorname{Inn}(L)$ in $G_{6}$ are

$$
\begin{aligned}
& A=\left\{g\left(f_{1}(k, l, m), k, f_{2}(k, l, m), f_{3}(k, l, m), l, m\right), k, l, m \in \mathbb{R}\right\}, \\
& B=\left\{g\left(h_{1}(u, v, w), u, h_{2}(u, v, w), h_{3}(u, v, w), v, w\right), u, v, w \in \mathbb{R}\right\},
\end{aligned}
$$

those to the group $\operatorname{Inn}(L)$ in $G_{i}, i=7,8$, are

$$
\begin{aligned}
& A=\left\{g\left(f_{1}(k, l, m), f_{2}(k, l, m), f_{3}(k, l, m), k, l, m\right), k, l, m \in \mathbb{R}\right\}, \\
& B=\left\{g\left(h_{1}(u, v, w), h_{2}(u, v, w), h_{3}(u, v, w), u, v, w\right), u, v, w \in \mathbb{R}\right\},
\end{aligned}
$$

where $f_{i}(k, l, m): \mathbb{R}^{3} \rightarrow \mathbb{R}$ and $h_{i}(u, v, w): \mathbb{R}^{3} \rightarrow \mathbb{R}, i=1,2,3$, are continuous functions with $f_{i}(0,0,0)=h_{i}(0,0,0)=0$. We prove that none of the groups $G_{i}$, $i=1, \ldots, 5$, and $G_{j}^{\varepsilon=1}, j=6,7,8$, satisfies the condition that for all $a \in A$ and $b \in B$ one has $a^{-1} b^{-1} a b \in \operatorname{Inn}(L)$. By Lemma 4 these groups are not multiplication groups of $L$. Taking the elements

$$
\begin{aligned}
a & =g\left(0, f_{1}(0,0, m), f_{2}(0,0, m), f_{3}(0,0, m), 0, m\right) \in A, \\
b & =g\left(0, h_{1}(0, v, 0), h_{2}(0, v, 0), h_{3}(0, v, 0), v, 0\right) \in B
\end{aligned}
$$

in $G_{i}, i=1,3,4,5$, the products $a^{-1} b^{-1} a b$ are contained in $\operatorname{Inn}(L)$ if and only if the equation

$$
\begin{aligned}
i=1: v m= & \left(1-e^{-m}\right) h_{2}(0, v, 0)+\left(e^{-v}-1\right) f_{3}(0,0, m) \\
& +h_{1}(0, v, 0) e^{-a v}\left(1-e^{-b m}\right)+f_{1}(0,0, m) e^{-b m}\left(e^{-a v}-1\right), \\
i=3:-v m= & \left(1-e^{-m}\right) h_{1}(0, v, 0)-v e^{-v} f_{2}(0,0, m) \\
& +\left(f_{3}(0,0, m)+\varepsilon f_{2}(0,0, m)\right)\left(e^{-v}-1\right),
\end{aligned}
$$




$$
\begin{aligned}
i=4:-v m= & h_{1}(0, v, 0) e^{-a v}\left(1-e^{-b m}\right)+f_{1}(0,0, m) e^{-b m}\left(e^{-a v}-1\right) \\
& +\left(1-e^{m}\right)\left(\varepsilon_{1} h_{2}(0, v, 0)+\varepsilon_{2} h_{3}(0, v, 0)\right) \\
& +(\cos v-1)\left(\varepsilon_{1} f_{2}(0,0, m)+\varepsilon_{2} f_{3}(0,0, m)\right) \\
& +\sin v\left(\varepsilon_{2} f_{2}(0,0, m)-\varepsilon_{1} f_{3}(0,0, m)\right) \\
i=5: v m= & \varepsilon_{1}\left[e^{-a v}\left(\cos (v) f_{2}(0,0, m)-\sin (v) f_{3}(0,0, m)\right)-f_{2}(0,0, m)\right] \\
& +\varepsilon_{2}\left[e^{-a v}\left(\sin (v) f_{2}(0,0, m)+\cos (v) f_{3}(0,0, m)\right)-f_{3}(0,0, m)\right] \\
& +h_{1}(0, v, 0)\left(1-e^{-m}\right)
\end{aligned}
$$

holds for all $m, v \in \mathbb{R}$. On the left hand side of (6), (7), (8), (9) there is the term $v m$ hence there does not exist any function $h_{i}(0, v, 0), f_{i}(0,0, m), i=1,2,3$, satisfying equations (6), (7), (8), (9).

Taking the elements $a=g\left(0, f_{1}(0,0, m), f_{2}(0,0, m), f_{3}(0,0, m), 0, m\right) \in A, b=$ $g\left(0, h_{1}(0, v, w), h_{2}(0, v, w), h_{3}(0, v, w), v, w\right) \in B$ of $G_{2}$ the products $a^{-1} b^{-1} a b$ are contained in $\operatorname{Inn}(L)$ if and only if the equation

$$
\begin{aligned}
m v= & e^{-m-w} f_{2}(0,0, m) v+h_{1}(0, v, w) e^{-a w-v}\left(1-e^{-a m}\right) \\
& +f_{1}(0,0, m) e^{-a m}\left(e^{-a w-v}-1\right) \\
& +\left(h_{3}(0, v, w)+\varepsilon h_{2}(0, v, w)\right) e^{-w}\left(1-e^{-m}\right) \\
& +\left(f_{3}(0,0, m)+\varepsilon f_{2}(0,0, m)\right) e^{-m}\left(e^{-w}-1\right)
\end{aligned}
$$

holds for all $m, v, w \in \mathbb{R}$. The left hand side is $m v$. But there does not exist any function $h_{i}(0, v, w), f_{i}(0,0, m), i=1,2,3$, satisfying equation (10).

Taking the elements $a=g\left(f_{1}(0,0, m), 0, f_{2}(0,0, m), f_{3}(0,0, m), 0, m\right) \in A, b=$ $g\left(h_{1}(0, v, 0), 0, h_{2}(0, v, 0), h_{3}(0, v, 0), v, 0\right) \in B$ of $G_{6}^{\varepsilon=1}$, respectively the elements

$$
\begin{aligned}
& a=g\left(f_{1}(0,0, m), f_{2}(0,0, m), f_{3}(0,0, m), 0,0, m\right) \in A, \\
& b=g\left(h_{1}(0, v, 0), h_{2}(0, v, 0), h_{3}(0, v, 0), 0, v, 0\right) \in B
\end{aligned}
$$

of $G_{7}^{\varepsilon=1, a=0}$ and of $G_{8}^{\varepsilon=1}$ the subgroup $\operatorname{Inn}(L)$ contains the products $a^{-1} b^{-1} a b$ if and only if in $G_{6}^{\varepsilon=1}$ the equation

$$
\begin{aligned}
\frac{1}{2} v^{2} m-v f_{1}(0,0, m)= & \left(1-e^{m}\right)\left(\varepsilon_{2} h_{2}(0, v, 0)+\varepsilon_{3} h_{3}(0, v, 0)\right)-\varepsilon_{1} v m \\
& +(\cos (v)-1)\left(\varepsilon_{2} f_{2}(0,0, m)+\varepsilon_{3} f_{3}(0,0, m)\right) \\
& +\sin (v)\left(\varepsilon_{3} f_{2}(0,0, m)-\varepsilon_{2} f_{3}(0,0, m)\right),
\end{aligned}
$$

respectively in $G_{7}^{\varepsilon=1, a=0}$ the equation

$$
\begin{aligned}
\frac{1}{2} v^{2} m-v f_{3}(0,0, m)= & \left(f_{1}(0,0, m)-h_{1}(0, v, 0)\right) e^{-v}\left(\varepsilon_{1} \cos (m)+\varepsilon_{2} \sin (m)\right) \\
& +\left(f_{2}(0,0, m)-h_{2}(0, v, 0)\right) e^{-v}\left(\varepsilon_{2} \cos (m)-\varepsilon_{1} \sin (m)\right) \\
& +\sin (m)\left(\varepsilon_{1} f_{2}(0,0, m)-\varepsilon_{2} f_{1}(0,0, m)\right) \\
& -\cos (m)\left(\varepsilon_{1} f_{1}(0,0, m)+\varepsilon_{2} f_{2}(0,0, m)\right) \\
& +e^{-v}\left(\varepsilon_{1} h_{1}(0, v, 0)+\varepsilon_{2} h_{2}(0, v, 0)\right)-\varepsilon_{3} v m,
\end{aligned}
$$


respectively in $G_{8}^{\varepsilon=1}$ the equation

$$
\begin{aligned}
\frac{1}{2} v^{2} m-v f_{3}(0,0, m)= & h_{1}(0, v, 0) e^{-v}\left(1-e^{-a m}\right)+h_{2}(0, v, 0)\left(1-e^{-m}\right) \\
& +f_{1}(0,0, m) e^{-a m}\left(e^{-v}-1\right)-\varepsilon_{1} v m
\end{aligned}
$$

holds for all $m, v \in \mathbb{R}$. On the left hand side of equations (11), (12), (13) there is the term $\frac{1}{2} v^{2} m$. Hence there does not exist any function $h_{i}(0, v, 0), f_{i}(0,0, m)$, $i=1,2,3$, satisfying equations (11), (12), (13).

The products $a^{-1} b^{-1} a b$ with $a=g\left(f_{1}(0, l, m), f_{2}(0, l, m), f_{3}(0, l, m), 0, l, m\right), b=$ $g\left(h_{1}(0, v, 0), h_{2}(0, v, 0), h_{3}(0, v, 0), 0, v, 0\right)$ in $G_{7}^{a \neq 0, \varepsilon=1}$ are contained in $\operatorname{Inn}(L)$ if and only if the equation

$$
\begin{aligned}
\frac{1}{2}\left(v l^{2}-\right. & \left.v^{2} l-a^{2} v m^{2}\right)+(a m+l) h_{3}(0, v, 0)-v f_{3}(0, l, m)-a m v^{2} \\
= & \varepsilon_{3} v a m+\left(f_{1}(0, l, m)-h_{1}(0, v, 0)\right) e^{-v-l}\left(\varepsilon_{1} \cos m+\varepsilon_{2} \sin m\right) \\
& +e^{-v} \varepsilon_{1} h_{1}(0, v, 0)+e^{-v} \varepsilon_{2} h_{2}(0, v, 0) \\
& +\left(f_{2}(0, l, m)-h_{2}(0, v, 0)\right) e^{-v-l}\left(\varepsilon_{2} \cos m-\varepsilon_{1} \sin m\right) \\
& +f_{2}(0, l, m) e^{-l}\left(\varepsilon_{1} \sin m+\varepsilon_{2} \cos m\right) \\
& -f_{1}(0, l, m) e^{-l}\left(\varepsilon_{2} \sin m+\varepsilon_{1} \cos m\right)
\end{aligned}
$$

holds for all $l, m, v \in \mathbb{R}$, where $\varepsilon_{i} \in\{0,1\}, i=1,2,3$, such that $\varepsilon_{1} \neq 0$ or $\varepsilon_{2} \neq 0$. Since on the left hand side of (14) there is the term $-\frac{1}{2} v^{2} l$ and $a \neq 0$ there does not exist any function $f_{i}(0, l, m), h_{i}(0, v, 0), i=1,2,3$, such that equation (14) holds.

The set

$$
\begin{aligned}
A_{1}=B_{1}= & \left\{g \left(e^{-m} \cos (l)-1, k, \frac{1}{\varepsilon_{2}^{2}+\varepsilon_{3}^{2}}\left(l e^{m}\left(\varepsilon_{2} \cos (l)-\varepsilon_{3} \sin (l)\right)+\sin (l)\right)\right.\right. \\
& \cdot\left(\varepsilon_{3} \cos (l)+\varepsilon_{2} \sin (l)\right), \frac{1}{\varepsilon_{2}^{2}+\varepsilon_{3}^{2}}\left(l e^{m}\left(\varepsilon_{2} \sin (l)+\varepsilon_{3} \cos (l)\right)\right. \\
& \left.\left.+\sin (l)\left(\varepsilon_{3} \sin (l)-\varepsilon_{2} \cos (l)\right), l, m\right) ; k, l, m \in \mathbb{R}\right\},
\end{aligned}
$$

with $\varepsilon_{2}, \varepsilon_{3} \in\{0,1\}$ and $\varepsilon_{2}^{2}+\varepsilon_{3}^{2} \neq 0$ is an $\operatorname{Inn}(L)_{6}$-connected left transversal in $G_{6}^{\varepsilon=0}$ which generates the group $G_{6}^{\varepsilon=0}$.

The set

$$
\begin{aligned}
A_{2}=B_{2}= & \left\{g \left(\frac{1}{\varepsilon_{1}^{2}+\varepsilon_{2}^{2}}\left(e^{l}(l+a m)\left(\varepsilon_{1} \cos (m)-\varepsilon_{2} \sin (m)\right)+\sin (m)\right)\right.\right. \\
& \cdot\left(\varepsilon_{1} \sin (m)+\varepsilon_{2} \cos (m)\right), \frac{1}{\varepsilon_{1}^{2}+\varepsilon_{2}^{2}}\left(e^{l}(l+a m)\left(\varepsilon_{1} \sin (m)+\varepsilon_{2} \cos (m)\right)\right. \\
& \left.\left.-\sin (m)\left(\varepsilon_{1} \cos (m)-\varepsilon_{2} \cos (m)\right), e^{-l} \cos (m)-1, k, l, m\right) ; k, l, m \in \mathbb{R}\right\},
\end{aligned}
$$

where $\varepsilon_{1}, \varepsilon_{2} \in\{0,1\}$ and $\varepsilon_{1}^{2}+\varepsilon_{2}^{2} \neq 0$ is an $\operatorname{Inn}(L)_{7}$-connected left transversal in $G_{7}^{\varepsilon=0}$ which generates the group $G_{7}^{\varepsilon=0}$.

The sets

$$
\begin{aligned}
& A=\left\{g\left(0, l e^{m}, 1-e^{-l-a m}, k, l, m\right) ; k, l, m \in \mathbb{R}\right\} \\
& B=\left\{g\left(-v e^{v+a w}, 0, e^{-w}-1, u, v, w\right) ; u, v, w \in \mathbb{R}\right\}
\end{aligned}
$$


are $\operatorname{Inn}(L)_{8}$-connected left transversals in the group $G_{8}^{\varepsilon=0}$ such that $A \cup B$ generates $G_{8}^{\varepsilon=0}$. This proves the assertion.

\section{References}

[A] A. A. Albert, Quasigroups I, Trans. Amer. Math. Soc. 54 (1943), 507-519.

[B1] R. H. Bruck, Contributions to the theory of loops, Trans. Amer. Math. Soc. 60 (1946), 245-354.

[B2] R. H. Bruck, A Survey of Binary Systems, Springer-Verlag, Berlin, 1971.

[C] P. Csörgö, M. Niemenmaa, On connected transversals to nonabelian subgroups, Europ. J. Comb. 23 (2002), 179-185.

[D] A. Drapal, Orbits of inner mapping groups, Monatsh. Math. 134 (2002), 191-206.

[F1] Á. Figula, The multiplication groups of 2-dimensional topological loops, J. Group Theory 12 (2009), 419-429.

[F2] Á. Figula, On the multiplication groups of three-dimensional topological loops, J. Lie Theory 21 (2011), 385-415.

[F3] Á. Figula, Three-dimensional topological loops with solvable multiplication groups, Comm. in Algebra 42 (2014), 444-468.

[F4] Á. Figula, Quasi-simple Lie groups as multiplication groups of topological loops, Adv. in Geometry 15 (2015), 315-331.

[F5] Á. Figula, M. Lattuca, Three-dimensional topological loops with nilpotent multiplication groups, J. Lie Theory 25 (2015), 787-805.

[F] R. Freese, R. McKenzie, Commutator Theory for Congruence Modular Varieties, London Mathematical Society Lecture Notes Series, Vol. 125, Cambridge University Press, Cambridge 1987.

[G] A. González-López, N. Kamran, P. J. Olver, Lie algebras of vector fields in the real plane, Proc. London Math. Soc. 64 (1992), no. 2, 339-368.

$[\mathrm{H}] \quad$ K. H. Hofmann, K. Strambach, Topological and analytical loops, in: Quasigroups and Loops: Theory and Applications, O. Chein, H. O. Pflugfelder, J. D. H. Smith eds., Heldermann-Verlag, Berlin, 1990, pp. 205-262.

[L] S. Lie, F. Engels, Theorie der Transformationsgruppen, Bd. 3. Verlag von B.G. Teubner, Leipzig, 1893.

[Ma] M. Mazur, Connected transversals to nilpotent groups, J. Group Theory 10 (2007), 195-203.

[Mo] C. Moore, D. Thérien, F. Lemieux, J. Berman, A. Drisko, Circuits and expressions with nonassociative gates, J. of Computer and System Sciences 60 (2000), 368394.

[Mu] Г. М. Мубаракзянов, Классификация разрешимых алгебр Ли шестого порядка с одним ненильпотентным базисным әлементом , Изв. вузов, Матем. 4 (1963), 104-116. [G. M. Mubarakzyanov, Classification of solvable Lie algebras in dimension six with one non-nilpotent basis element, Izv. Vyssh. Uchebn. Zaved. Mat. 4 (1963), 104-116 (in Russian)].

[NV] G. P. Nagy, P. Vojtěchovský, Moufang loops with commuting inner mappings, J. Pure Appl. Algebra 213 (2009), 2177-2186. 
[N] P. T. Nagy, Nuclear properties of loop extensions, Results in Math. 74 (2019), Art. number: 100 (2019).

[NS1] P. T. Nagy, K. Strambach, Loops in Group Theory and Lie Theory, Walter de Gruyter, Vol. 35, Berlin, 2002.

[NS2] P. T. Nagy, K. Strambach, Schreier loops, Czech. Math. J. 58 (2008), 759-786.

[NK1] M. Niemenmaa, T. Kepka, On multiplication groups of loops, J. Algebra 135 (1990), 112-122.

[NK2] M. Niemenmaa, T. Kepka, On connected transversals to abelian subgroups, Bull. Austral. Math. Soc. 49 (1994), 121-128.

[RT] M. Rawashdeh, G. Thompson, The inverse problem for six-dimensional codimension two nilradical Lie algebras, J. Math. Phys. 47 (2006), 112901-112929.

[ST] A. Shabanskaya, G. Thompson, Six-dimensional Lie algebras with a five-dimensional nilradical, J. of Lie Theory 23 (2013), 313-355.

[SV1] D. Stanovský, P. Vojtěchovský, Commutator theory for loops, J. Algebra 399 (2014), 290-322.

[SV2] D. Stanovský, P. Vojtěchovský, Abelian Extensions and Solvable Loops, Results. Math. 66 (2014), 367-384.

[T] P. Turkowski, Solvable Lie algebras of dimension six, J. Math. Phys. 31 (1990), 1344-1350.

[V] A. Vesanen, Solvable loops and groups, J. Algebra 180 (1996), 862-876.

Funding Information Open Access funding provided by University of Debrecen.

Open Access This article is licensed under a Creative Commons Attribution 4.0 International License, which permits use, sharing, adaptation, distribution and reproduction in any medium or format, as long as you give appropriate credit to the original author(s) and the source, provide a link to the Creative Commons licence, and indicate if changes were made. The images or other third party material in this article are included in the article's Creative Commons licence, unless indicated otherwise in a credit line to the material. If material is not included in the article's Creative Commons licence and your intended use is not permitted by statutory regulation or exceeds the permitted use, you will need to obtain permission directly from the copyright holder. To view a copy of this licence, visit http://creativecommons.org/ licenses/by/4.0/.

Publishers Note Springer Nature remains neutral with regard to jurisdictional claims in published maps and institutional affiliations. 